\title{
Report of the APS Neutrino Study Reactor Working Group
}

E. Abouzaid, K. Anderson, G. Barenboim, B. Berger, E. Blucher, T. Bolton,

S. Choubey, J. Conrad, J. Formaggio, S. Freedman, D. Finley, P. Fisher,

B. Fujikawa, M. Gai, M. Goodman, A. de Gouvea, N. Hadley, R. Hahn, G. Horton-Smith, R. Kadel, B. Kayser, K. Heeger, J. Klein, J. Learned, M. Lindner, J. Link, K.-B. Luk, R. McKeown, I. Mocioiu, R. Mohapatra, D. Naples, J. Peng, S. Petcov, J. Pilcher, P. Rapidis, D. Reyna, M. Shaevitz, R. Shrock, N. Stanton, R. Stefanski, R. Yamamoto, M. Worcester

October 28,2004 


\section{Contents}

1 Introduction $\quad 1$

2 A Reactor Experiment to Measure $\sin ^{2} 2 \theta_{13} \quad 2$

2.1 Strategies to Improve on Past Reactor Neutrino Oscillation Experiments . . 3

2.2 Classes of $\theta_{13}$ Reactor Neutrino Oscillation Experiments _ . . . . . . . 10

2.2.1 Small: Sensitivity of $\sin ^{2} 2 \theta_{13} \sim 0.03 \ldots \ldots \ldots$. . . . . . . 12

2.2.2 Medium: Sensitivity of $\sin ^{2} 2 \theta_{13} \sim 0.01 \ldots \ldots \ldots \ldots$

2.2.3 Large: Sensitivity of $\sin ^{2} 2 \theta_{13} \leq 0.005$ - The Ultimate $\theta_{13}$ Experiment? 13

2.3 Time Scale, Costs, and the Global Situation for $\theta_{13}$ Experiment . . . . . 13

3 Comparisons and Combinations of Reactor and Accelerator Neutrino Oscillation Experiments $\quad 15$

3.1 Introduction . . . . . . . . . . . . . . . . . 15

3.2 Determination of $\theta_{13} \ldots \ldots \ldots \ldots \ldots$

3.3 Resolution of the $\theta_{23}$ Degeneracy . . . . . . . . . . . . . 22

3.4 Constraining CP Violation Parameters . . . . . . . . . . . . 22

3.5 Determining the Mass Hierarchy . . . . . . . . . . . . . . 29

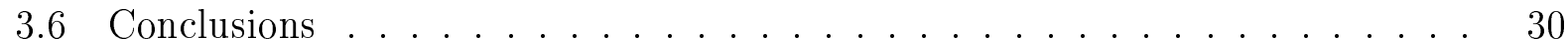

4 Beyond $\theta_{13} \quad 30$

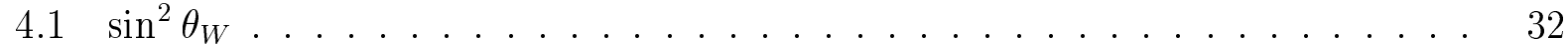

4.2 Precision Measurement of $\theta_{12}$ with a Second Generation Long Baseline Reactor

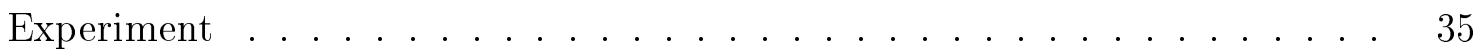

4.2.1 Physics Motivation . . . . . . . . . . . . . 35

4.2.2 Long Baseline Reactor Experiments . . . . . . . . . . . . . . 36

4.2 .3 Potential Experiment . . . . . . . . . . . . 38

4.3 Sterile Neutrinos . . . . . . . . . . . . . . . 40

4.4 Tests of CPT Symmetry . . . . . . . . . . . . . . . . 41

4.5 Supernova Neutrinos . . . . . . . . . . . . . . . . 41

4.6 Massive Anti-Neutrino Detectors for Monitoring Worldwide Reactors . . . 42

4.7 A Possible Reactor at the Center of the Earth . . . . . . . . . . . . . 43 
5 Conclusion 


\section{Executive Summary}

The worldwide program to understand neutrino oscillations and determine the meutrino mixing parameters, $\mathrm{CP}$ violating effects, and mass hierarchy will require a broad combination of measurements. Our group believes that a key element of this future neutrino program is a multi-detector neutrino experiment (with baselines of $\sim 200 \mathrm{~m}$ and $\sim 1.5 \mathrm{~km}$ ) with a sensitivity of $\sin ^{2} 2 \theta_{13}=0.01$. In addition to oscillation physics, the reactor experiment may provide interesting measurements of $\sin ^{2} \theta_{W}$ at $Q^{2}=0$, neutrino couplings, magnetic moments, and mixing with sterile neutrino states.

$\theta_{13}$ is one of the twenty-six parameters of the standard model, the best model of electroweak interactions for energies below $100 \mathrm{GeV}$ and, as such, is worthy of a precision measurement independent of other considerations. A reactor experiment of the proposed sensitivity will allow a measurement of $\theta_{13}$ with no ambiguities and significantly better precision than any other proposed experiment, or will set limits indicating the scale of future experiments required to make progress. Figure 1 shows a comparison of the sensitivity of reactor experiments of different scales with accelerator experiments for setting limits on $\sin ^{2} 2 \theta_{13}$ if the mixing angle is very small, or for making a measurement of $\sin ^{2} 2 \theta_{13}$ if the angle is observable. A reactor experiment with a $1 \%$ precision may also resolve the degeneracy in the $\theta_{23}$ parameter when combined with long-baseline accelerator experiments (see Fig. 2).

In combination with long-baseline measurements, a reactor experiment may give early indications of $\mathrm{CP}$ violation and the mass hierarchy. The combination of the T2K and Nova long-baseline experiments will be able to make significant measurements of these effects if $\sin ^{2} 2 \theta_{13}>0.05$ and with enhanced beam rates can improve their reach to the $\sin ^{2} 2 \theta_{13}>0.02$ level. If $\theta_{13}$ turns out to be smaller than these values, one will need other strategies for getting to the physics. Thus, an unambiguous reactor measurement of $\theta_{13}$ is an important ingredient in planning the strategy for the future neutrino program.

\section{Recommendations:}

Our group has one highest priority recommendation:

- We recommend the rapid construction of a multi-detector reactor experiment with a sensitivity of 0.01 for $\sin ^{2} 2 \theta_{13}$.

Our other recommendations are the following:

- To help accomplish our highest priority recommendation, we recommend R\&D support necessary to prepare a full proposal.

- We recommend continued support for the KAMLAND experiment. KAMLAND has made the best determination of $\Delta m_{12}^{2}$ to date, and will provide the best measurement 

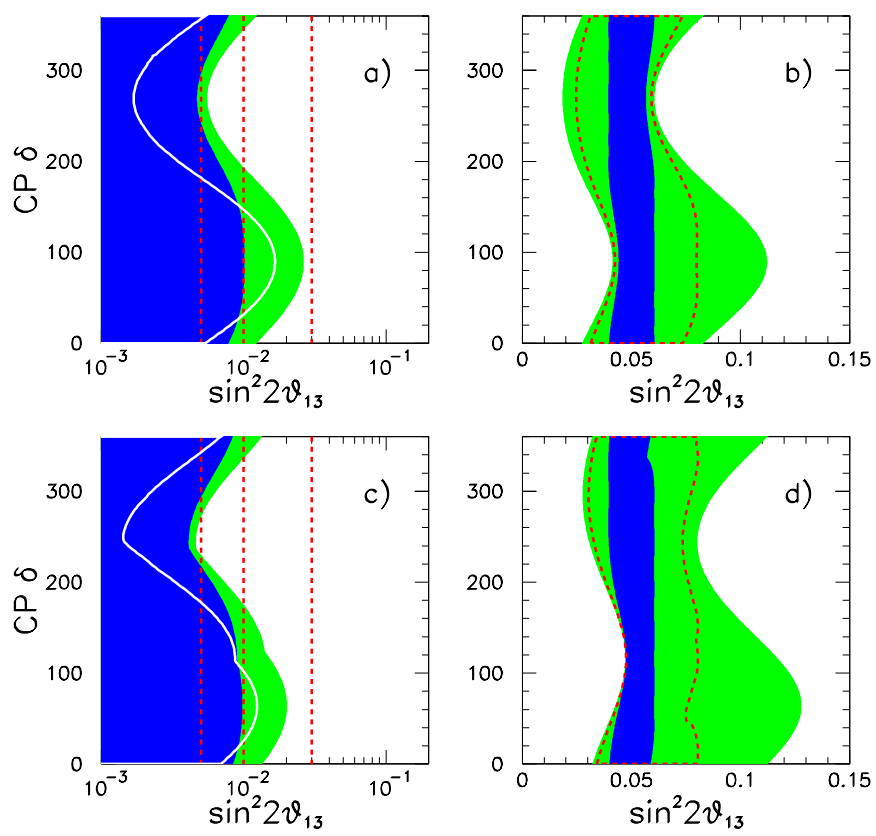

Figure 1: $90 \%$ C.L. regions and upper limits for various oscillation measurements for (a,c) $\sin ^{2} 2 \theta_{13}=0$ and (b,d) $\sin ^{2} 2 \theta_{13}=0.05$. The top (bottom) plots are for the T2K (Nova) long-baseline experiments. The three vertical dashed lines correspond to the $90 \%$ C.L. upper limits of $0.005,0.01$, and 0.03 possible with different scales of reactor experiments. The green region (white curve) is the $90 \%$ C.L. allowed region for the two long-baseline experiments for a five year neutrino-only run with nominal $(\times 5)$ beam rate and the blue region gives the combination of the five year long-baseline and medium reactor measurements.

for the foreseeable future. As the deepest running reactor experiment, it also provides critical information about cosmic-ray related backgrounds for future experiments.

- We recommend the exploration of potential sites for a next-generation experiment at a distance of $70 \mathrm{~km}$ from an isolated reactor complex to make high precision measurements of $\theta_{12}$ and $\Delta m_{12}^{2}$.

- We recommend support for development of future large-scale reactor $\theta_{13}$ experiments that fully exploit energy spectrum information. 

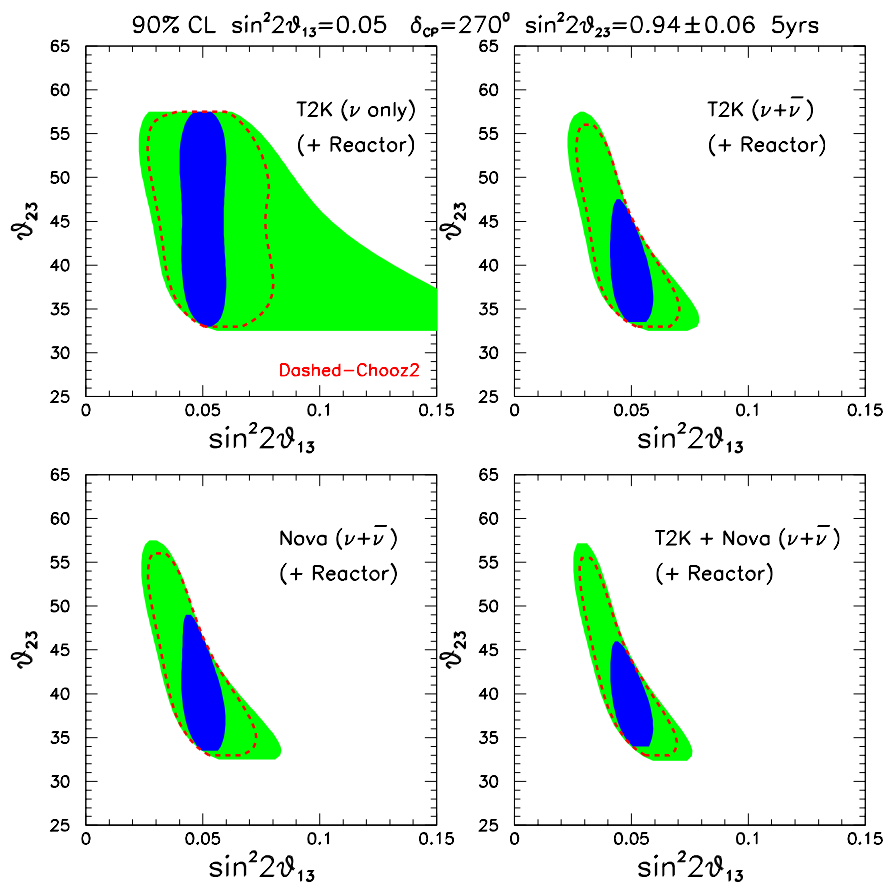

Figure 2: $90 \%$ C.L. allowed regions for simulated data with an underlying oscillation parameters of $\sin ^{2} 2 \theta_{13}=0.05, \theta_{23}=38^{0}, \Delta m^{2}=2.5 \times 10^{-3} \mathrm{eV}^{2}$ and $\delta_{C P}=270^{\circ}$. The analysis includes the restriction that $\sin ^{2} 2 \theta_{23}=0.94 \pm 0.06$. The green regions are for various combinations of the T2K and/or Nova experiments for five years of running periods. The blue regions are the $90 \%$ C.L. allowed regions for the combination of a reactor experiment with sensitivity of $\sin ^{2} 2 \theta_{13}=0.01$ and a long-baseline experiment. The dashed red lines show how the combined measurement would degraded with a reactor experiment with 3 times worse sensitivity. 


\section{Introduction}

During recent years, we have learned a great deal about the neutrino sector. Nuclear reactors have played a critical role in this work, from the first direct detection of neutrino by Reines and Cowan to recent measurements at Kamland, Chooz, and Palo Verde.

Future goals of the $\nu$ program include making precise measurements of the mixing angles of the MNSP matrix, resolving the mass hierarchy, and searching for CP violation in neutrino oscillations. These last measurements, however, are not independent, and share one common feature: the need of some knowledge of the "connecting" angle, $\theta_{13}$. This angle couples (or decouples) the solar and atmospheric oscillations and opens (or closes) the door to the study of $\mathrm{CP}$ violation in the leptonic sector $\left(\theta_{13}=0\right.$ will render the $\mathrm{CP}$ phase non-physical and therefore make nonviable an explanation of the matter-antimatter asymmetry of the Universe as a result of leptogenesis through the Dirac phase). The importance of its measurement cannot be overestimated.

On the other hand, the value of $\theta_{13}$ is one of the yet unknown parameters of the Standard Model (together with the absolute neutrino mass and the scalar mass), and as such is of the utmost importance if a complete theory of flavor is to be achieved.

Our working group has studied the potential role of experiments at nuclear reactors in the future neutrino program. Most attention has been focused on a measurement of $\theta_{13}$, which plays a central role in resolving many open questions in neutrino physics. As will be described, we believe that a 2-detector reactor experiment with a sensitivity of $\sin ^{2} 2 \theta_{13} \sim$ 0.01 can play a critical role in the future neutrino program. Such an experiment, unlike appearance experiments, does not suffer from parameter degeneracies (or ambiguities), The short baseline also means that it is free of matter effects. A reactor experiment therefore provides the only clean and self-contained measurement of $\theta_{13}$.

In addition to providing information about $\theta_{13}$, a reactor experiment, when combined with accelerator experiments, may give early indications of CP violation and the mass hierarchy, and can be the only way to resolve the degeneracy in $\theta_{23}$, something accelerator experiments (ever when multiple experiments are combined) cannot do.

A reactor experiment may also provide a different measurement of the Weinberg angle, $\sin ^{2} \theta_{W}$, at $Q^{2}=0$ (assuming that the Standard Model is correct), or help to test the magnitude and nature of the neutrino neutral current coupling. It is important to remember that not only is this coupling rather poorly known, but such a new measurement may shed light on the $\mathrm{NuTeV}$ anomaly. It may turn out that a reactor measurement of $\sin ^{2} \theta_{W}$ will play a crucial role in exploring new physics effects.

Reactor experiments will also provide a goldmine of useful information on neutrino couplings, magnetic moments, mixing with sterile neutrino states, CPT in the neutrino sector, 
and supernova physics.

This report is divided into three main sections: a discussion of an experiment to measure $\theta_{13}$ at a nuclear reactor, a study of the physics impact of reactor experiments of different sensitivity, and a discussion of other physics topics that may be addressed by experiments using nuclear reactors as a neutrino source.

\section{A Reactor Experiment to Measure $\sin ^{2} 2 \theta_{13}$}

The best current limit on $\sin ^{2} 2 \theta_{13}$ comes from the CHOOZ experiment [1]. This experiment had a single, 5-ton fiducial mass detector at a baseline of $1.05 \mathrm{~km}$ from a $8.9 \mathrm{GW}$ (thermal), two-reactor plant. Figure 3 shows their exclusion region on a plot of $\Delta m_{13}^{2}$ vs. $\sin ^{2} 2 \theta_{13}$. After approximately one year of datataking with the reactors running, they found the measuredto-expected flux ratio to be $(1.01 \pm 2.8 \pm 2.7) \%$. The $2.7 \%$ systemtatic error had two principal sources: the reactor neutrino flux $(2 \%)$ and the absolute detector acceptance (1.5\%). It is worth noting that there is no systematic error from background, because CHOOZ was able to do a direct background subtraction based on extensive data taking during reactor-off time; a direct background subtraction likely will not be possible in future experiments at sites with multiple reactor cores.

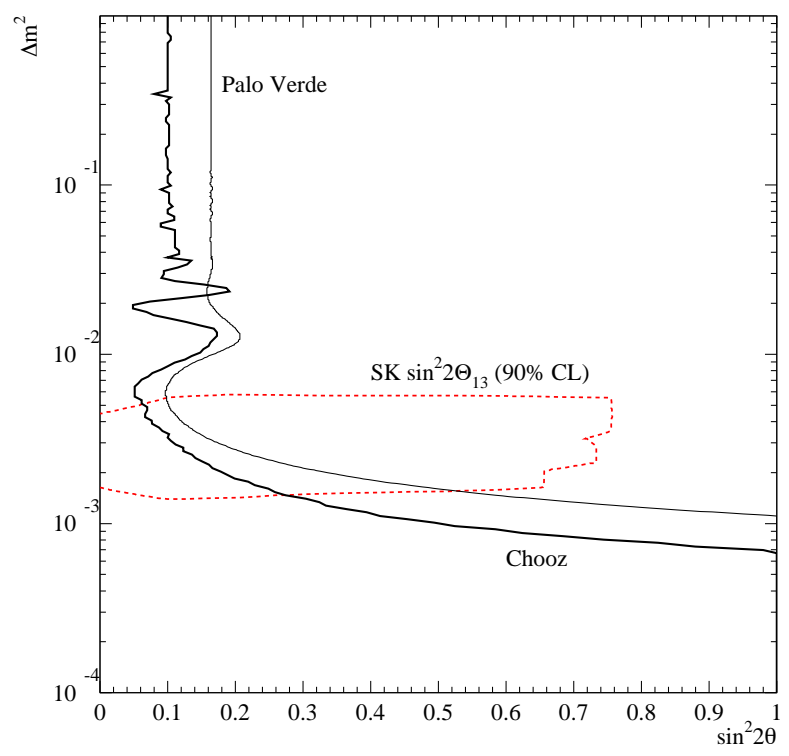

Figure 3: Plot showing regions of $\theta_{13}$ and $\Delta m^{2}$ excluded by CHOOZ and Palo Verde experiments (to the right of solid curves). Figure from Ref. [2].

To improve on the sensitivity of the CHOOZ experiment, it will be necessary to reduce 
both statistical and systematic uncertainties. Two main classes of systematic uncertainty in the CHOOZ experiment may be reduced by adding an additional detector close to the reactor. A near detector largely eliminates the reactor flux uncertainty. It also simplifies somewhat the acceptance calculation, requiring only an understanding of the relative acceptance of two detectors rather than the absolute acceptance of a single detector. In addition, future experiments can be optimized to collect higher statistics, to reduce backgrounds, to be closer to the optimal $L / E$ based on recent $\Delta m_{13}^{2}$ measurements. The relative position of the near and far detector will determine the experiment's sensitivity to changes in the observed $\bar{\nu}_{e}$ interaction rate and spectral distortion due to neutrino oscillation.

The discovery and measurement of a non-zero $\theta_{13}$ will require an unambigous oscillation signal. In the presence of neutrino oscillations the $\bar{\nu}_{e}$ rate and energy spectra measured in detectors at different distances from a reactor will be different. From the difference in the observed rate of $\bar{\nu}_{e}$ interactions we can deduce the amount of neutrino mixing assuming that all systematic effects are under control. An observation of energy-dependent changes in the measured spectrum is a unique signature of neutrino oscillations and can be used to discover and measure the oscillation effect. Depending on the size and optimization a next-generation reactor experiment can make use of both the rate and spectral information to measure $\theta_{13}$

In Section 2.1, we review the different elements of optimizing a multiple detector $\theta_{13}$ reactor experiment. Section 2.2 discusses parameters of future experiments needed to attain different levels of sensitivity.

\subsection{Strategies to Improve on Past Reactor Neutrino Oscillation Experiments}

\section{Experimental Layout and Baseline Optimization:}

The layout of the experiment and the optimum distances for the detectors is determined by the oscillation frequency in the $(1,3)$ channel which depends on the mass splitting $\Delta m_{13}=\Delta m_{12}+\Delta m_{23} \simeq \Delta m_{23}$ for $\Delta m_{12}^{2} \simeq 10^{-5} \mathrm{eV}^{2}$ and $\Delta m_{23}^{2} \simeq 10^{-3} \mathrm{eV}^{2}$. Hence, the frequency of the $(1,3)$ oscillation and the distances of the detectors will primarily depend on the atmospheric mass splitting $\Delta m_{23}^{2}$. For $\Delta m_{23}^{2} \simeq 2 \times 10^{-3} \mathrm{eV}^{2}$ and a mean energy of $E_{\bar{\nu}_{e}}=$ $3.5 \mathrm{MeV}$ the oscillation wavelength in the $(1,3)$ channel is $\sim 4 \mathrm{~km}$. The first minimum in the survival probability occurs at a distance of $\sim 2 \mathrm{~km}$ from a reactor. The simplest approach to measuring $\theta_{13}$ is to use the first detector as a near detector to normalize the $\bar{\nu}_{e}$ flux from the reactor while the second detector - also referred to as the far detector - is placed at a distance that maximizes its sensitivity to a suppression in the $\bar{\nu}_{e}$ interaction rate and/or a distortion

in the observed $\bar{\nu}_{e}$ spectrum. This distance corresponds approximately to the position of the first minimum in the survival probability. For small to medium-sized detectors the statistical significance of the oscillation signal is dominated by the difference in the interaction rate in 
the near and far detectors, however, the observation of a relative distortion in the observed spectra can provide a unique signature of the $(1,3)$ oscillation. In general, for a rate-based two-detector experiment an optimal distance of $\sim 1.7 \mathrm{~km}$ for the far detector is found [3]. This optimum is sensitive to the value of $\Delta m^{2}$. Details of such optimizations are discussed in $[3,4]$. It is possible to think of other detector configurations that are optimized to measure differences in the energy spectrum between the near and far detectors, rather than to make the most precise rate measurements. These approaches require much larger detectors.

\section{Statistical Precision and Target Volume:}

In the past, reactor neutrino experiments, with a total volume ranging from a few tons (CHOOZ) to $1 \mathrm{kt}$ (KamLAND), have been built. The large size of KamLAND is required to collect sufficient event statistics from neutrino sources averaging $180 \mathrm{~km}$ away. Future reactor neutrino experiment, to measure $\theta_{13}$ at a baseline of $\mathcal{O}(1 \mathrm{~km})$, will require event statistics in excess of $\sim 30,000$ events in the far detector to reach a statistical error of $\leq 0.5 \%$. A search

for spectral distortions will require even larger event rates. For a rate-based $\theta_{13}$ reactor experiment detector sizes of 25-50 $\mathrm{t}$ are needed and for a shape measurement a fiducial volume in excess of $100 \mathrm{t}$ is required. The use of a powerful reactor complex with multiple cores will increase the event statistics. Most modern two core systems have thermal power ranging from 5-8 GW-thermal. While multi-core systems help with the event rate they eliminate the possibility of a direct background measurement during a reactor-off period. The CHOOZ experiment [1] used an extended period of reactor off running to determine and subtract a $9.5 \%$ background, presumably due to muon-induced spallation backgrounds. Future reactor neutrino experiments are unlikely to have this capability. Figure 4 shows the expected number of events as a function of distance and volume for a CHOOZ-like reactor complex (assuming no oscillations).

\section{Reducing Source Systematics: Reactor Flux Uncertainty:}

A relative measurement between multiple detectors will largely eliminate the error in the reactor $\bar{\nu}_{e}$ flux. The reactor $\bar{\nu}_{e}$ flux is predicted from the thermal power of the reactor and the known spectra of the isotopes involved in the neutrino producing fission processes. The total number of reactor antineutrinos and their energy spectrum can be calculated to about $2 \%$ and $2.5 \%$ respectively. This error enters any absolute flux measurement that compares the observed $\bar{\nu}_{e}$ interaction rate in the detector to the predicted reactor $\bar{\nu}_{e}$ flux. In a relative measurement this uncertainty is reduced to $\leq 0.2 \%$. At multi-core sites the residual error comes from the superposition of $\bar{\nu}_{e}$ fluxes at different distances to the detectors.

Reducing Detector Systematics: An Optimized Detector Design: Most detector designs for a next-generation $\theta_{13}$ experiment are based rather closely on the CHOOZ design, consisting of a central region of Gd-loaded liquid scintillator surrounded by some buffer regions. Anti-neutrino interactions are detected by the inverse beta decay reaction, $\bar{\nu}_{e}+p \rightarrow$ $e^{+} n$, followed by the signal from neutron capture in the Gd-loaded scintillator ( $8 \mathrm{MeV}$ 


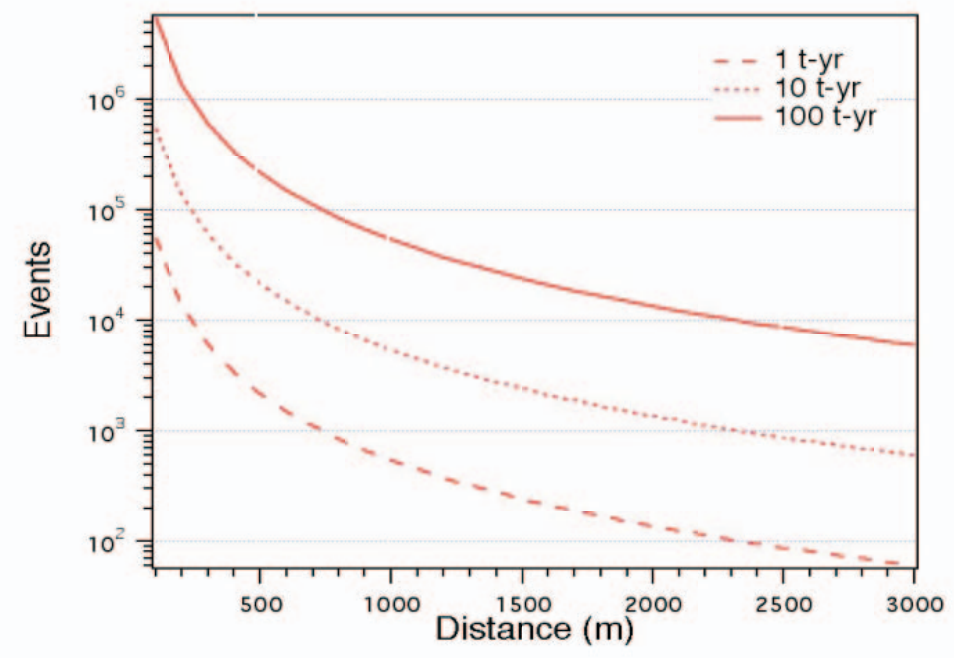

Figure 4: Expected number of events in a CHOOZ-like detector at a reactor complex with a thermal power of $6.5 \mathrm{GW}$.

energy release in photons). Event selection may be based only on the timing and energies of the two signals, without any requirement on reconstructed event positions. The energy of the neutron capture events is well understood and can together with spallation events from cosmic-ray muons be used for the relative energy calibration of the detectors. Eliminating cuts on the reconstructed position of events is crucial for reaching an accuracy of better than $1 \%$ on the total anti-neutrino interaction rate.

\section{Calibration of Relative Detector Acceptance:}

The concept of using multiple detectors for a relative measurement of the $\bar{\nu}_{e}$ interaction rate requires a very good understanding of the relative acceptance of the two detectors. Several issues determine the relative detector acceptance of a multi-detector system:

\section{1. fiducial volume}

2. neutron detection efficiency (in presence of backgrounds)

3. relative energy scale (for spectrum measurement)

4. accidental and correlated backgrounds

In general, it will be necessary to understand the efficiency of $\bar{\nu}_{e}$ detection as a function of energy, event position in the target volume, detector, and detector position with respect to the reactor. For mountainous underground sites the detector position determines the 
overburden and shielding from cosmic rays as well as the natural background around the detectors.

Several strategies and techniques have been proposed to understand the relative detector acceptance. They are summarized in Table 2. A multi-layer detector with a Gd-doped inner liquid scintillator volume surrounded by a scintillating, gamma catcher region and a mineral oil buffer, allows the detection of the $\bar{\nu}_{e}$ coincidence signal without a fiducial volume or energy threshold cut. This eliminates two important sources of systematic error. Cross calibration of detectors using the large flux of $\bar{\nu}_{e}$ at the near detector site, address all source of normalization and acceptance errors. This approach, which requires that the detectors be movable, presents logistical and technical challenges. One concern is that the detector response itself may change during this operation and make it impossible to determine the relative detector acceptance without absolute calibrations before and after the move.

Preliminary studies show that a combination of the tabulated techniques may lead to an effective systematic error of $\sim 0.8 \%$ in the calibration of the relative detector acceptance [4], while a cross calibration approach could push the relative acceptance error down to $\leq 0.4 \%$ [5].

While many of the systematic issues we expect to encounter in a precision $\theta_{13}$ reactor neutrino experiment have been studied in the previous CHOOZ, Palo Verde, and KamLAND experiments, there may be additional experimental challenges in the quest to push the sensitivity. A redundant set of calibrations and techniques to determine the relative detector acceptance are required. It will be necessary to design an experimental program that eliminates the possibility of a "single-point failure" in the measurement, the characterization of the detector response, and the analysis of the data. In light of these considerations a future reactor neutrino experiment should have

1. an extensive calibration program to determine the absolute detector response over the $\bar{\nu}_{e}$ energy range

2. redundant strategies for mitigating the systematic error in the relative measurement including the cross calibration of the detectors.

Only an experiment with redundant methods to deal with systematic errors will be able to achieve the ambitious design goal of a sensitivity of $\sin ^{2} 2 \theta_{13} \leq 0.01$. For rate and shape based measurements most of the systematic error sources are identical. In a shape-based measurement, however, the absolute calibration of the energy scale and its linearity will require special study.

\section{Improving the Signal-to-Noise Ratio: Background Reduction}

Accidental and correlated backgrounds have presented a serious challenge to all past reactor neutrino experiments. In the CHOOZ experiment a 9.5\% background [1] measured 
during the reactor-off time was subtracted from the candidate event set. This allowed the CHOOZ experiment to obtain an absolute precision of $2.7 \%$ on the reactor $\bar{\nu}_{e}$ flux measurement. This background was most likely due to muon-induced spallation products and accidentals in the lower energy region of the spectrum. Future reactor neutrino experiments are likely to be located at reactor complexes with multiple cores (to increase the event statistics) and won't have the ability to make an independent measurement of these backgrounds with all of the reactors off. While careful material selection and detector design can reduce the accidental backgrounds, muon-induced spallation backgrounds are best reduced by going deep underground. Scaling the observed number of backgrounds in the CHOOZ experiment to the requirements for a future experiment we obtain the signal to muon-related background ratio for a spherical liquid scintillator detector as a function of overburden (see Figure 5). A further reduction in the background rate may be achieved by the use of more extensive veto systems.

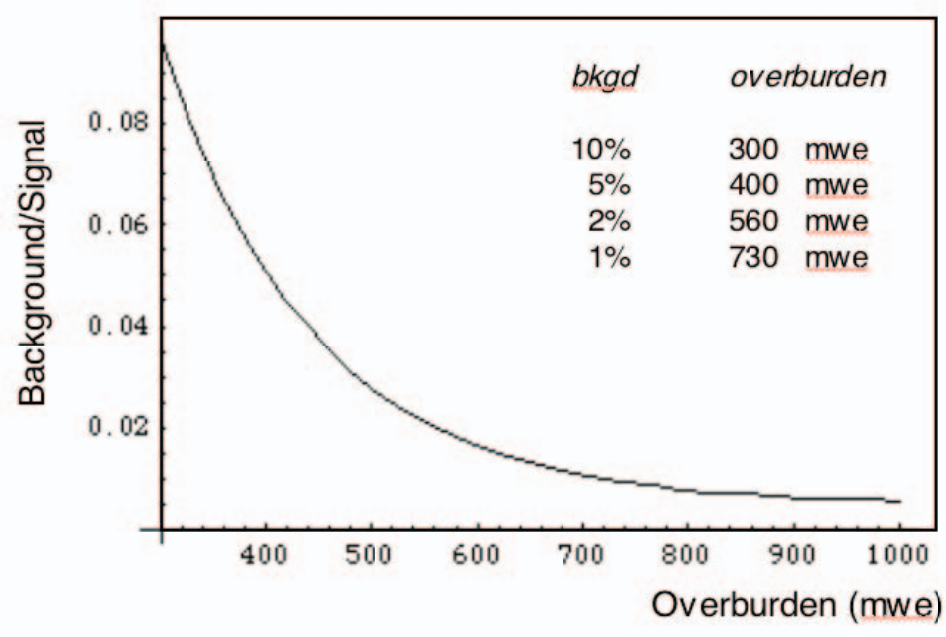

Figure 5: Signal to background ratio in a typical spherical detector of a next-generation reactor neutrino experiment for a CHOOZ-like mountainous underground detector site.

Muons produce spallation backgrounds in the inner detector and fast neutrons in the rock that form a correlated background to the $\bar{\nu}_{e}$ candidate event signal. Correlated backgrounds are one of the principal challenges in a precision reactor neutrino experiment. Below we provide a comparison of the expected muon-related backgrounds in two typical mediumsized experiments at the Braidwood and Daya Bay reactor sites (see Section 2.2.2).

A high-efficiency, extended muon tracking system surrounding the liquid scintillator can be used to tag muons that enter the detector and pass through rock and shielding in the vicinity of the target. The purpose of such a muon tracking system is two-fold: (1) It can add to the effective depth of the detector by vetoing muons and (2) help understand the rate 
and spectral shape of correlated backgrounds by tagging muon-related backgrounds. The effective signal to background ratio depends on both the overburden as well as muon rejection and can only be determined from detailed simulations for a specific experiment. Even with an effective veto, it is desirable to place the detectors as deep as possible to minimize the dead time associated with veto of muon-related backgrounds in the detector.

The results of $[6,7,8]$ are used to calculate the muon flux, neutron flux, and isotope production in underground liquid scintillators at mountainous and flat sites. The simulation corresponds to a good approximation to the topography at the Daya Bay reactor complex and the Braidwood power plant. Table 1 shows the results for three different geometries, a flat geometry at 450 mwe and two different configurations with mountains. The muon flux at the far detector for the simulated mountain is about 8 times lower than the flat geometry and the neutron flux and isotope production are lower by a factor of 6 . This is due to the energy dependence of the isotope production. Figures $6 \mathrm{a}$ and $6 \mathrm{~b}$ show the results of the muon and neutron flux calculations.

\section{A Reactor Experiment in Phases: Homogeneous versus modular detectors:}

The detector design requires the choice between a homogeneous, single-detector concept (like CHOOZ and KamLAND) or a modular detector system. Single detectors minimize the surface area for intrinsic backgrounds relative to the fiducial volume. At a potentially higher cost, modular detectors have the advantage of increased mobility and easier calibration and testing. In contrast to a single, large detector a modular system can be built and evaluated in the underground facility prior to the completion of the entire detector volume. Modular detectors also create a scalable system that can be expanded to reach the physics goals. For example, one can imagine a phased approach that would allow the construction of a timely medium-scale experiment to make a rate-based search for $\theta_{13}$ while providing the possibility of expansion for a shape-based, precision measurement of $\theta_{13}$, once it has been shown to be non-zero.

Table 1: Comparison of neutron rates and cosmogenics for three different geometries. The flat site corresponds to the parameters of the Braidwood site, the mountains with 230 and 1100 mwe are a good approximation to the overburden at Daya Bay.

\begin{tabular}{|l|l|l|l|}
\hline & $\begin{array}{l}\text { flat, } \\
450 \text { mwe }\end{array}$ & $\begin{array}{l}\text { mountain, } \\
230 \text { mwe }\end{array}$ & $\begin{array}{l}\text { mountain, } \\
1100 \text { mwe }\end{array}$ \\
\hline muon flux $\left(\mathrm{m}^{-2} \mathrm{~S}^{-1}\right)$ & 0.194 & 1.63 & 0.024 \\
neutron rate $\left(\right.$ ton $\left.^{-1} \mathrm{day}^{-1}\right)$ & 161 & 824 & 30.0 \\
${ }^{8} \mathrm{He}+{ }^{9} \mathrm{Li}\left(\right.$ ton $\left.^{-1} \mathrm{day}^{-1}\right)$ & $0.076 \pm 0.026$ & $0.4 \pm 0.4$ & $0.014 \pm 0.002$ \\
\hline
\end{tabular}



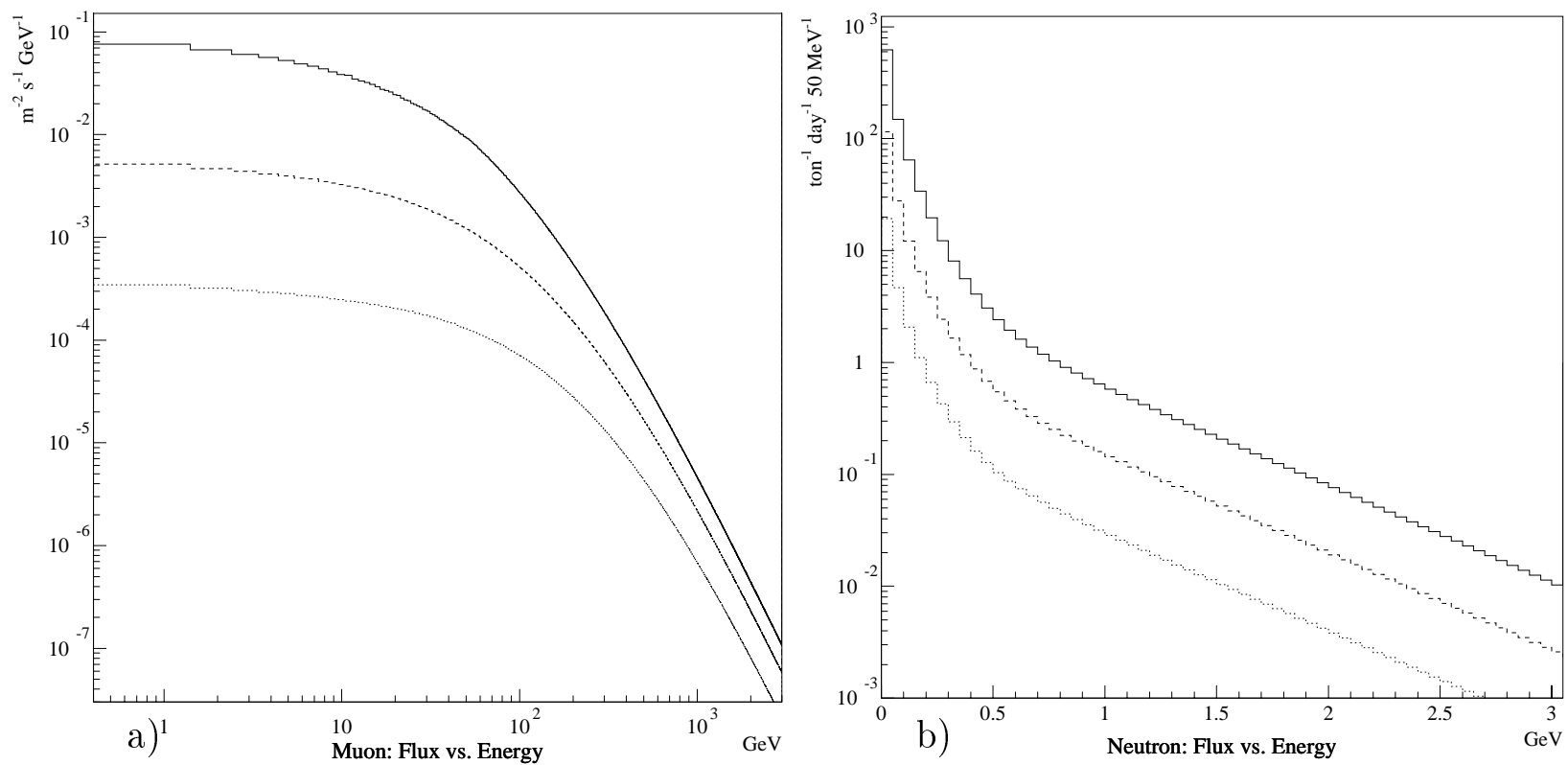

Figure 6: a) Muon flux and b) neutron flux as a function of energy computed for three different geometries. The upper curve is the flux for the near detector in a mountain with 230 mwe overburden, the middle curve for 450 mwe in a flat geometry, and the lower curve is for a detector at vertical depth of 1100 mwe in a mountain configuration similar to Daya Bay. 
Over the course of the past year, several groups have proposed a variety of detector concepts. The design and size of these concepts depend largely on constraints imposed by the underground laboratory. A generic optimization of the detector has not yet been done. However, it appears that a multi-layer detector with a Gd-doped liquid scintillator target may be the design concept of choice to make a measurement without fiducial volume and energy cuts.

Table 2: Methods for the relative calibration of detector acceptance.

\begin{tabular}{|l|l|}
\hline \hline Issue & Method \\
\hline target volume & $\begin{array}{l}\text { flow and weight measurements } \\
\text { multi-layer scintillator detector, } \\
\text { no fiducial volume cut in Gd-scintillator } \\
\text { cross calibration with movable detectors }\end{array}$ \\
neutron detection efficiency & $\begin{array}{l}\text { cross calibration with movable detectors } \\
\text { source calibration }\end{array}$ \\
energy scale & $\begin{array}{l}\text { extensive calibrations with sources } \\
\text { relative calibration with neutron-capture peak } \\
\text { cross calibration with movable detectors }\end{array}$ \\
geometrical detector effects & identical design \\
\hline
\end{tabular}

In summary, Table 3 shows the principal contributions to the systematic error in a nextgeneration reactor neutrino oscillation experiment to measure $\theta_{13}$ :

\subsection{Classes of $\theta_{13}$ Reactor Neutrino Oscillation Experiments}

In this section, we describe scales of experiments needed to reach three different levels of sensitivity: $\sin ^{2} 2 \theta_{13} \sim 0.03$ (referred to as small), $\sin ^{2} 2 \theta_{13} \sim 0.01$ (medium), and $\sin ^{2} 2 \theta_{13} \sim$ 0.005 (large). For each class, we consider experiments designed to reach these sensitivities at $90 \%$ confidence level after 3 years of data collection.

Using generic assumptions about the detector design and systematic errors, the ultimate $\theta_{13}$ sensitivity of various, simple 2-detector systems has been studied by Huber et al. [3, 9]. Figure 7 gives an indication of the magnitude of the detector size and precision needed to reach a specific sensitivity in $\sin ^{2} 2 \theta_{13}$. 
Table 3: Projected systematic error budget in a next-generation reactor neutrino experiment.

\begin{tabular}{|l|l|l|}
\hline Effect & Error Estimate & Method \\
\hline reactor flux uncertainty & $\leq 0.2 \%$ & relative measurement at different distances \\
$\begin{array}{l}\text { detection efficiency \& } \\
\text { target volume }\end{array}$ & $0.8 \%$ to $\leq 0.4 \%$ & $\begin{array}{l}\text { calibration of relative det. efficiency, } \\
\text { no fiducial volume cut in Gd scintillator, } \\
\text { flow and weight measurement of target }\end{array}$ \\
backgrounds & $1.0 \%$ to $<0.5 \%$ & sufficient overburden, active and passive shielding \\
\hline Total Systematics & $1 \%$ to $0.5 \%$ & \\
\hline
\end{tabular}
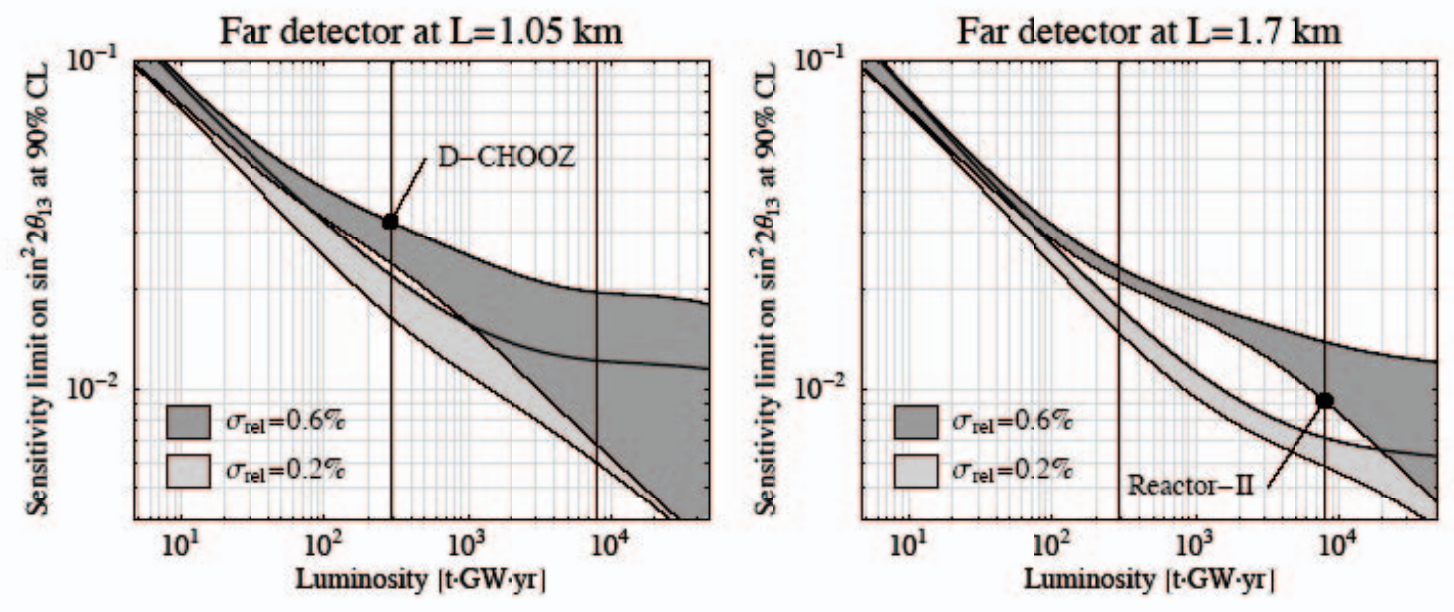

Figure 7: Sensitivity of reactor neutrino experiments to $\sin ^{2} 2 \theta_{13}$ as a function of the integrated luminosity. Figure adapted from [3]. The left panel shows the projected sensitivity of the Double-CHOOZ experiment, a small-scale experiment at a distance of $1.05 \mathrm{~km}$. The right panel shows the sensitivity of medium or large scale experiments. Figure from Ref. [9]. 


\subsubsection{Small: Sensitivity of $\sin ^{2} 2 \theta_{13} \sim 0.03$}

An example of a small-scale experiment is Double-CHOOZ [13]. The Double-CHOOZ Collaboration [13] has proposed a new, two-detector experiment with sensitivity of $\sin ^{2} 2 \theta_{13} \sim 0.03$ at the site of the old CHOOZ experiment. The experiment will use detectors with 10 ton fiducial mass, twice the mass of the original CHOOZ detector. They will reuse the existing underground lab at a distance of $1.05 \mathrm{~km}$. Although the non-optimal distance limits their sensitivity, particularly for lower $\Delta m^{2}$ values, it eliminates the need for civil construction at the far site. The near detector for the Double-CHOOZ experiment will be placed at a modest depth of 5-10 m underground with additional man-made overburden of up to 50 mwe. The shallow depth of the near detector may lead to significant dead time but keeps the overall civil construction for this project low.

The sensitivity of Double-CHOOZ and other "small" reactor experiments comes mainly from a precise measurement of the total $\bar{\nu}_{e}$ interaction rate. Limited statistics make it difficult to observe the energy dependence of the oscillation.

\subsubsection{Medium: Sensitivity of $\sin ^{2} 2 \theta_{13} \sim 0.01$}

Concepts for an oscillation experiment with a sensitivity of $\sin ^{2} 2 \theta \leq 0.01$ are being studied at several reactor sites. US groups have led the investigation at Braidwood in Illinois, the Diablo Canyon in California, the Angra dos Reos in Brazil and the Daya Bay in China [10, 11]. In comparison to small reactor $\theta_{13}$ experiments, the designs usually include larger detectors (25100 tons), located in deeper (230-1200 mwe) underground laboratories at optimized distances from the reactor, with reduced backgrounds, and integrated calibration and muon tracking systems. In comparison to the small-scale experiment with a sensitivity goal of $\sin ^{2} 2 \theta_{13} \sim$ 0.03, medium-sized experiments aim to be large enough to provide precise information on the $\bar{\nu}_{e}$ interaction rate and spectrum to reach an experimental sensitivity of $\sin ^{2} 2 \theta_{13} \leq$ 0.01. This requires improvements and optimization in the detector design as well as the experimental layout. Perhaps most importantly, sufficient overburden and active background reduction are required to achieve a sensitivity of $1 \%$. Proposals for medium-scale experiments usually offer the option of movable detectors to allow a direct cross-check of the relative detector acceptance. Multiple detector modules also provide the opportunity for additional consistency checks.

The basic experimental parameters of the different proposals with US involvement are summarized in Table 4. 
Table 4: Basic design parameters of the proposed $\theta_{13}$ reactor experiments.

\begin{tabular}{|l|l|l|l|l|l|}
\hline Proposal & $\begin{array}{l}\text { Baseline } \\
(\text { Near/Far })\end{array}$ & $\begin{array}{l}\text { Overburden } \\
(\text { Near } / F a r)\end{array}$ & $\begin{array}{l}\text { Detector Size } \\
(\text { Near } / F a r)\end{array}$ & Sensitivity $\left(\sin ^{2} 2 \theta_{13}\right)$ & Ref. \\
\hline Double CHOOZ & $0.2 / 1.05$ & $50 / 300$ mwe & $10 / 10 \mathrm{t}$ & 0.03 & {$[13]$} \\
Braidwood & $0.2 / 1.7$ & $450 / 450$ mwe & $130 / 130 \mathrm{t}$ & 0.01 & {$[10]$} \\
Diablo Canyon & $0.4 / 1.7$ & $150 / 750$ mwe & $50 / 100 \mathrm{t}$ & 0.01 & {$[11]$} \\
Angra, Brazil & $0.3 / 1.5$ & $200 / 1700$ mwe & $50 / 500 \mathrm{t}$ & 0.01 & {$[12]$} \\
Daya Bay, China & $0.3 / 1.8-2.2$ & $300 / 1100$ mwe & $50 / 100 \mathrm{t}$ & 0.01 & \\
\hline
\end{tabular}

\subsubsection{Large: Sensitivity of $\sin ^{2} 2 \theta_{13} \leq 0.005$ - The Ultimate $\theta_{13}$ Experiment?}

Although many challenges will have to be overcome to reach a precision of $\sin ^{2} 2 \theta_{13} \sim$ 0.01 , it is possible that significantly larger experiments could ultimately be used to make the most precise measurement of the mixing angle. Experiments with fiducial mass of $>$ 100 tons can precisely measure the difference in energy spectra between the near and far detectors, providing a measurement of $\sin ^{2} 2 \theta_{13}$ that is independent of knowledge of the relative normalization of the detectors. This method is sensitive, of course, to the relative understanding of energy reconstruction and the energy dependence of background in the two detectors. Furthermore, a multi-detector setup with three or more detectors has the potential to maximize the experiment's statistical sensitivity to both the rate and shape difference, and may verify the oscillation signal at different baselines. While it is not practical to consider an experiment with a truly continuous, variable baseline we can entertain the possibility of having multiple detector halls at different baselines to map out the survival probability.

We envision that a large reactor neutrino oscillation experiment could be built in a second phase to a successful medium-sized modular experiment that discovers non-zero $\theta_{13}$.

\subsection{Time Scale, Costs, and the Global Situation for $\theta_{13}$ Experi- ment}

Critical to all efforts to measure $\theta_{13}$ with reactor neutrinos is an underground laboratory to shield the antineutrino detectors from cosmic rays and cosmic ray muon-induced spallation products that can mimic the coincidence structure of $\bar{\nu}_{e}$ interactions. This requirement is common to all proposals for a future reactor neutrino oscillation experiment. The overburden requirement for the $\bar{\nu}_{e}$ detectors varies depending on the power of the reactor, the distance of the detector from the reactor, and details of the detector design. In any experiment 
the effective signal to background ratio depends critically on the amount of overburden, the topology of the site, and the muon veto and tracking systems. Unless an existing underground laboratory can be found at the right distance from a nuclear power plant significant civil construction efforts are necessary to create the environment to perform this experiment. Conceptual design studies by several groups have investigated the feasibility of constructing vertical shafts into the ground or horizontal tunnels into mountains to obtain the required overburden. The results of these preliminary investigations are summarized in the White Paper of the International $\theta_{13}$ Working Group [4].

Several US groups are involved in the international effort to find a suitable power plant for this experiment and the design effort. The Braidwood and Diablo Canyon site in the US, Daya Bay in China, Angro dos Reos in Brazil,and Chooz in France are being investigated as possible options. Braidwood and Diablo Canyon would offer the opportunity for a USbased experiment. An experiment at Daya Bay would be based on a partnership between the Institute of High Energy Physics (IHEP) in Beiing and the US, and a project at Angra would be based on a partership between US and Brazilian institutions. A Letter of Intent has been prepared for the Double-Chooz experiment in France and some US groups are interested in joining.

For a greenfield site in the US the civil construction costs for the underground laboratory are estimated to be of the order of $\$ 20-30 \mathrm{M}$. This is comparable to the costs for three mediumsized 25-ton detectors. An evaluation of two possible reactor sites (Braidwood in Illinois and Diablo Canyon in California) suggest that the construction of a suitable underground laboratory will take about 18-36 months depending on the type of excavation necessary and method used. We expect that the civil construction at the two sites under investigation in the US will take about two years. The time scale and possible milestones for a US-based experiment are shown in Figure 8.

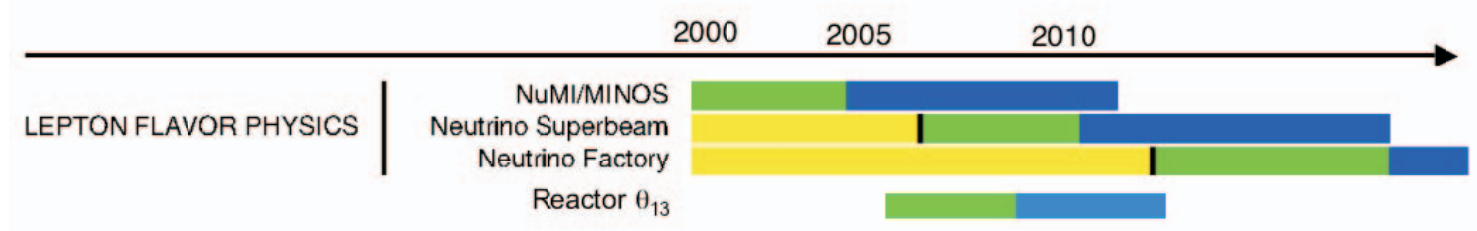

Figure 8: Projected time scale and roadmap for a $\theta_{13}$ reactor experiment in the US.

Besides the US-led studies at Braidwood and Diablo Canyon a R\&D partnership has been formed between several institutions in the US and IHEP to study the possibility of a future reactor neutrino experiment at the Daya Bay nuclear power plant near Hong Kong. In this case the US groups would likely be responsible for the design and construction of the antineutrino detectors, or subsystems thereof, while the civil construction of the underground 
laboratory would be undertaken by IHEP and other institutions in China.

A European collaboration has proposed the Double-CHOOZ experiment. This proposal intends to make use of the existing underground laboratory near the Chooz nuclear power plant for the far detector and to build an additional near detector next to the power plant. The project received scientific approval in France in March 2004, and a collaboration consisting of groups in France, Italy, Germany, and Russia has been formed. Construction of the far detector can commence as early as 2006 assuming that the respective funding agencies in France and Germany will approve the proposal in 2004/2005. Together with EDF (Electricite de France), the Double-Chooz collaboration is studying the possibility of building an underground covern at a depth of $\sim 8 \mathrm{~m}$ with additional overburden in form of a man-made mountain. The cost for this construction is likely to be covered by EDF. Without the civil construction cost for the near detector the Double-Chooz experiment is estimated at about Euro 10M. First results from both the near and far detector can be expected in 2008/2009. With this ambitious time scale the Double-Chooz experiment is likely to set the time scale for the worldwide search for $\theta_{13}$.

\section{Comparisons and Combinations of Reactor and Ac- celerator Neutrino Oscillation Experiments}

\subsection{Introduction}

The worldwide program to understand neutrino oscillations and determine the mixing parameters, CP violating effects, and mass hierarchy will require a broad combination of measurements. Progress in the past associated with solving the solar and atmospheric neutrino puzzles took a full suite of experiments to isolate and understand the phenomenology. As measurements became available, they defined the direction for future studies. One would expect a similar chain for the current goals where the program grows as information is obtained. This study attempts to see how various present proposals for next generation experiments (including two detector reactor and accelerator-based, long-baseline experiments) compare and can complement each other. A particular emphasis is on combining experiments to give improved physics parameter determination. As in the past, the best constraints on the phenomenology come from combining data from various processes and setups.

For a three active neutrino scenario, neutrino oscillations are described by six physics parameters: $\theta_{13}, \theta_{12}, \theta_{23}, \Delta m_{12}^{2}, \Delta m_{23}^{2}$, and the CP violation phase, $\delta$. In addition, a full description also requires knowing the hierarchy of mass state 3 relative to 1 and 2, i.e. the sign of $\Delta m_{23}^{2}$. Of the six parameters, it is assumed for this study that $\theta_{12}, \theta_{23}, \Delta m_{12}^{2}$, and $\Delta m_{23}^{2}$ are known to the precision expected from either the current program (SuperK, Minos 
Table 5: Current and future uncertainty estimates on oscillation parameters. This study assumes values corresponding to the future estimates.

\begin{tabular}{|cc|c|c|}
\hline Parameter & Value & Current $\sigma$ & Future $\sigma$ \\
\hline $\sin ^{2} 2 \theta_{23}$ & 1.0 & $0.06($ SuperK $)$ & $0.01(\mathrm{~T} 2 \mathrm{~K})$ \\
$\Delta m_{23}^{2}\left(\mathrm{eV}^{2}\right)$ & $2.5 \times 10^{-3}$ & $0.33 \times 10^{-3}($ SuperK $)$ & $0.05 \times 10^{-3}(\mathrm{~T} 2 \mathrm{~K})$ \\
$\theta_{12}(\mathrm{deg})$ & 30 & - & - \\
$\Delta m_{12}^{2}\left(\mathrm{eV}^{2}\right)$ & $7.1 \times 10^{-5}$ & - & - \\
\hline
\end{tabular}

and CNGS) or the future program (Nova and T2K). This leaves for determination $\theta_{13}, \delta$, and the mass hierarchy which are the subject of this study. Table 5 lists the values as well as the current and future errors used in the study for $\theta_{12}, \theta_{23}, \Delta m_{12}^{2}$, and $\Delta m_{23}^{2}$.

The other experimental inputs for the study are given in Table 6 and are derived from estimates of the measurement sensitivities. Three reactor experiments are considered corresponding to a small (Double-CHOOZ), medium(Braidwood, Daya Bay type), or large (MiniBooNE size) reactor $\bar{\nu}_{e}$ measurement. Two offaxis long-baseline experiments are considered, JPARC to SuperK (T2K) and the NuMI offaxis proposal (Nova). The sensitivities for the reactor experiments are scaled from the $\sin ^{2} 2 \theta_{13} 90 \%$ C.L. limits at $\Delta m_{23}^{2}=2.5 \times 10^{-3} \mathrm{eV}^{2}$ for a null oscillation scenario. For the long-baseline experiments, the uncertainties are scaled from the expected number of events given in the Nova proposal and a recent talk by Y. Suzuki at the Seesaw workshop. The given uncertainties include statistical errors associated with the background and signal for a 5 year data run but no systematic uncertainty.

The uncertainty on the $\theta_{23}$ parameter can have a significant effect on the long-baseline measurements since the quantity that is constrained as given in Table 5 is $\sin ^{2} 2 \theta_{23}$ and the parameter that modulates the long-baseline oscillation probability is $\sin ^{2} \theta_{23}$. This can lead to a $65 \%(23 \%)$ uncertainty in the oscillation probability with the present (future) errors.

For the studies given below, the uncertainties due to the variations of $\theta_{23}, \Delta m_{23}^{2}$, and the mass hierarchy are included. For $\bar{\nu}$ running, there is a $5 \%$ to $20 \%$ contamination of $\nu$ oscillation events, but, for these studies, this contamination is assumed to be zero. Results are typically given for five year data runs with nominal beam rates but in addition some results are presented for a five-fold increased rate which would somewhat correspond to an upgraded long-baseline program with a new proton driver at Fermilab or the upgrade at JPARC.

\subsection{Determination of $\theta_{13}$}

The $\theta_{13}$ mixing angle is one of the big unknowns in the current neutrino oscillation phenomenology. It appears to be somewhat smaller than the other mixing angles. The size of 
Table 6: Estimates of the experimental uncertainties associated with various future oscillation experiments. For the long-baseline experiments, the given uncertainties include statistical errors associated with the background and signal for a 5 year data run but no systematic uncertainty.

\begin{tabular}{|c|c|c|c|c|}
\hline \multirow[b]{2}{*}{ Experiment } & \multirow{2}{*}{$\begin{array}{l}\text { Basis of } \\
\text { Estimate }\end{array}$} & \multicolumn{3}{|c|}{ Osc. Prob. and $\sigma$ for $\sin ^{2} 2 \theta_{13}=$} \\
\hline & & 0.02 & 0.05 & 0.10 \\
\hline $\begin{array}{r}\text { Reactor }\left(E_{\nu}=3.6 \mathrm{MeV}\right) \\
\langle L\rangle\end{array}$ & $\begin{array}{l}\sin ^{2} 2 \theta_{13}^{\text {Limit }} \\
@ \Delta m_{23}^{2}=2.5 \times 10^{-3} \mathrm{eV}^{2}\end{array}$ & & & \\
\hline Small $1.05 \mathrm{~km}$ & $0.03 @ 90 \% C L$ & $0.013 \pm 0.018$ & $0.032 \pm 0.018$ & $0.064 \pm 0.018$ \\
\hline Medium & $0.01 @ 90 \% C L$ & $0.020 \pm 0.006$ & $0.050 \pm 0.006$ & $0.100 \pm 0.006$ \\
\hline $1.8 \mathrm{~km}$ & $0.005 @ 90 \% C L$ & $0.020 \pm 0.003$ & $0.050 \pm 0.003$ & $0.100 \pm 0.003$ \\
\hline $\begin{array}{l}\text { Nova }\left(E_{\nu}=2.3 \mathrm{GeV}\right) \\
\langle L\rangle=810 \mathrm{~km}\end{array}$ & $\begin{array}{l}\mathrm{N}_{\text {events }}^{\text {5yrs }}: \sin ^{2} 2 \theta_{13}=0.1, \delta_{C P}=0 \\
\text { @ } \Delta m_{23}^{2}=2.5 \times 10^{-3} \mathrm{eV}^{2}\end{array}$ & & & \\
\hline$\nu$ & 175.2 signal / 38.1 bkgnd & $0.011 \pm 0.002$ & $0.025 \pm 0.003$ & $0.048 \pm 0.003$ \\
\hline $\bar{\nu}$ & 66 signal / 22 bkgnd & $0.008 \pm 0.003$ & $0.018 \pm 0.004$ & $0.034 \pm 0.005$ \\
\hline
\end{tabular}

$\theta_{13}$ is an important ingredient in constraining models of neutrino masses and mixing including attempts to relate the quark and lepton mixings. The size of this mixing angle also has important implications for long-baseline $\nu_{e}$ appearance measurements because it scales the size of the oscillation probability.

The leading order dependence of the oscillation probability for reactor and long-baseline measurements is given by

$$
\begin{aligned}
P_{\text {reactor }} & =1-P\left(\nu_{e} \rightarrow \nu_{e}\right) \simeq \sin ^{2} 2 \theta_{13} \sin ^{2}\left(1.27 \Delta m_{31}^{2} L / E\right) \\
P_{\text {long-baseline }} & =P\left(\nu_{\mu} \rightarrow \nu_{e}\right) \simeq \sin ^{2} 2 \theta_{13} \sin ^{2} \theta_{23} \sin ^{2}\left(1.27 \Delta m_{31}^{2} L / E\right) \\
& + \text { Other terms with CP viol. and matter effects }
\end{aligned}
$$

In the investigations shown here, the full formulae for the oscillation probability have been used as incorporated in a computer program developed by Stephen Parke. The higher order corrections for the reactor probability are quite small for the above distances and a measurement of $P_{\text {reactor }}$ is simply related to a constraint on the mixing parameter $\theta_{13}$. On the other hand, the full expression for the long-baseline probability introduces many degeneracies and correlations between the physics parameters $\theta_{23}$ and $\delta_{C P}$ plus the mass hierarchy through matter effects even before experimental uncertainties are taken into account. Therefore, a measurement of $P_{\text {long-baseline }}$ corresponds to regions in the physics parameter space.

As an indication of how well a given measurement can constrain the value of $\theta_{13}$, Figure 9 (top: T2K, bottom: Nova) shows the $90 \%$ C.L. allowed regions associated with measurements 
of a null oscillation scenario where $\sin ^{2} 2 \theta_{13}=0$. The three vertical dashed lines correspond to the $90 \%$ C.L. upper limits from a large, medium, and small reactor measurement as presented in Table 6. The green region (white curve) is the $90 \%$ C.L. allowed region for the two long-baseline experiments for a five year neutrino only run with the nominal $(\times 5)$ beam rate. Combining the long-baseline and medium reactor measurement gives the improved blue region. In all cases, the results include the variations associated with the uncertainty in $\theta_{23}, \Delta m_{23}^{2}$, and mass hierarchy.

If $\theta_{13}$ is large enough, then positive signals will be observed by the experiments. Under these circumstances, the goal would be to make the best determination of the mixing parameter. Figure 10 shows the $90 \%$ C.L. regions that will be obtained for an underlying scenario where $\sin ^{2} 2 \theta_{13}=0.05$. As shown, a long-baseline only measurement will not determine the mixing parameter $\theta_{13}$ very well with an allowed region that spans from 0.02 to over 0.11 . On the other hand, a reactor experiment with at least the medium scale sensitivity measures $\sin ^{2} 2 \theta_{13}$ to about $10 \%$ and $\theta_{13}$ to $\pm 0.4^{\circ}$.

As seen from the figures, the reactor measurements are very efficient at constraining the value of $\theta_{13}$ and even a small reactor experiment can probe for an early indication that the value is sizeable. The large reactor experiment has sensitivity comparable to planned long-baseline experiments and the medium scale experiment can measure values in the range for $\sin ^{2} 2 \theta_{13}>0.02$ at the $10 \%$ to $20 \%$ level. As will be seen in later plots, studies of CP violation and matter effects over the next decade are only possible if $\sin ^{2} 2 \theta_{13}$ is significantly larger than about 0.01 . A small or medium scale reactor experiment can set the scale if these studies will be possible and, if they are, add additional information for the determining the parameters.

As outlined above, $\theta_{13}$ is a key parameter both for oscillation physics and for planning the experimental program. For this reason, it is important for the near term program to make as precise and robust a measurement as possible including cross checks of the experimental results. In addition, measuring $\theta_{13}$ using different processes, such as $\bar{\nu}_{e}$ disappearance and $\nu_{\mu} \rightarrow \nu_{e}$ appearance, is a test of both the experimental procedure and also the physics model. In the same way that overconstraining the CKM with various measurements is a prime tool for testing the Standard Model, these redundant measurements could point to problems with the neutrino physics phenomenology. Figure 11 shows an example scenario where a medium scale reactor measurement differs from the results of $\mathrm{T} 2 \mathrm{~K}$ and Nova due to an additional high $\Delta m^{2}$ component such as would be associated with a positive MiniBooNE/LSND signal. For the figure, the primary oscillation signal is associated with $\sin ^{2} 2 \theta_{13}=0.02$ and $\delta=180^{\circ}$, and the added MiniBooNE component has an oscillation probability of $1 \%$ for both neutrinos and antineutrinos for the T2K and Nova setups. To isolate the oscillation physics in a case such as this will require a combined analysis using the data from all the measurements, MiniBooNE, reactor, and long-baseline. 

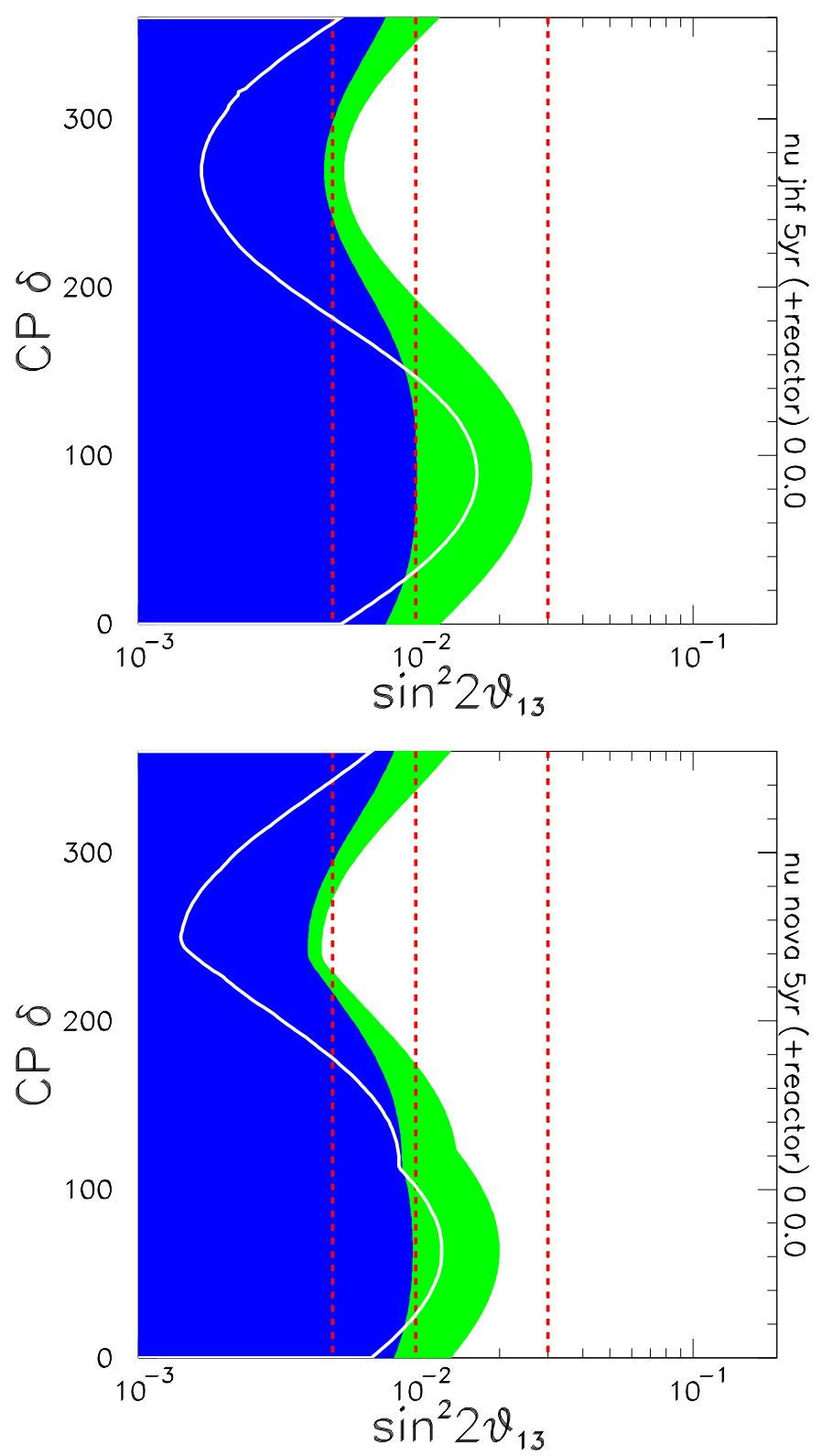

Figure 9: 90\% C.L. regions and upper limits for various oscillation measurements for an underlying null oscillation scenrio where $\sin ^{2} 2 \theta_{13}=0$. The top (bottom) plot is for the T2K (Nova) long-baseline experiments. The three vertical dashed lines correspond to the $90 \%$ C.L. upper limits from a large, medium, and small reactor measurement as presented in Table 6. The green region (white curve) is the $90 \%$ C.L. allowed region for the two long-baseline experiments for a five year neutrino only run with nominal $(\times 5)$ beam rate and the blue region gives the combination of the five year long-baseline and medium reactor measurements. 

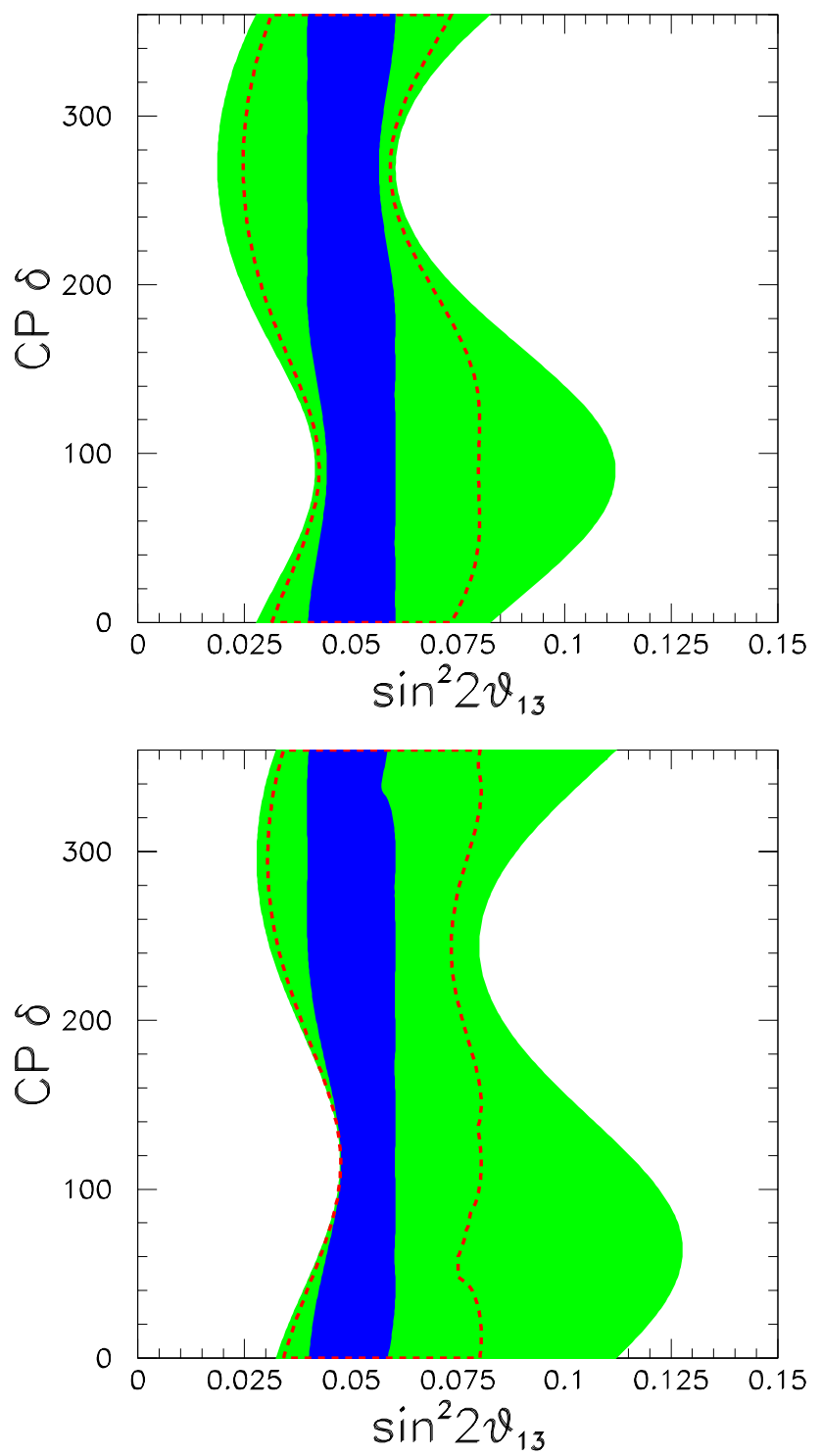

Figure 10: $90 \%$ C.L. regions for underlying oscillation parameters of $\sin ^{2} 2 \theta_{13}=0.05, \Delta m^{2}=$ $2.5 \times 10^{-3} \mathrm{eV}^{2}$ and $\delta_{C P}=0$. The green regions are for the T2K (top plot) or Nova (bottom plot) experiments for five years of neutrino running. The blue regions are the 90\% C.L. allowed regions for a combined medium reactor plus long-baseline analysis. The dashed red lines indicate how the combined measurement would degrade with the small reactor sensitivity. 


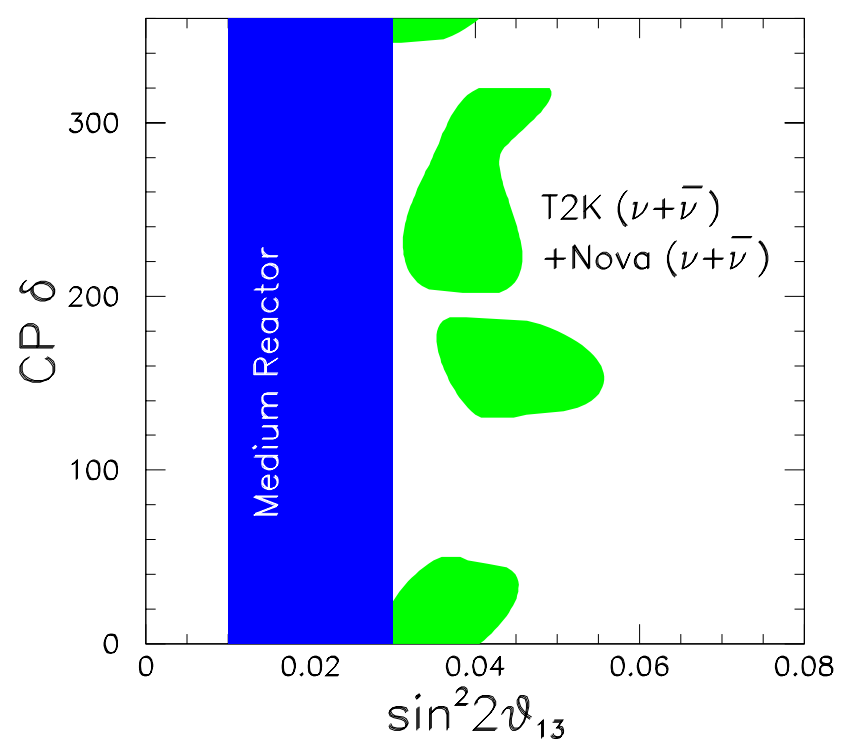

Figure 11: Example of reactor and long-baseline measurements that show a discrepancy due to an extra MiniBooNE signal in the long-baseline data. The primary oscillation signal is associated with a $\sin ^{2} 2 \theta_{13}=0.02$ and $\delta=180^{\circ}$. An additional high $\Delta m^{2}$ MiniBooNE component has been added with an oscillation probability of $1 \%$ for both neutrinos and antineutrinos. The long-baseline results are for five years runs of neutrinos and antineutrinos for both T2K and Nova. 


\subsection{Resolution of the $\theta_{23}$ Degeneracy}

The mixing angle $\theta_{23}$ is an important parameter in developing an understanding of the mixing matrix and for proceeding with a determination of $\theta_{13}$. In many theoretical models, $\theta_{23}$ is not expected to be $45^{\circ}$ and the difference from this value,both in sign and magnitude, may lead to a deeper understanding of the mixing.

Information on the value of $\theta_{23}$ has been obtained from $\nu_{\mu}$ disappearance measurements in the atmospheric $\Delta m^{2}$ region, for example from the Super-K and K2K experiments . These experiments restrict the allowed region for the quantity $\sin ^{2} 2 \theta_{23}$. Unfortunately, a value for

$\sin ^{2} 2 \theta_{23}=a$ corresponds to two possible values for $\theta_{23}, \frac{1}{2} \sin ^{-1}(\sqrt{a})$ or $\frac{\pi}{2}-\frac{1}{2} \sin ^{-1}(\sqrt{a})$. The current Super-K measurement of $\sin ^{2} 2 \theta_{23}=1.00 \pm 0.06$ corresponds to values of $\theta_{23}=$ $45^{0} \pm 7^{0}$. For the determination of $\theta_{13}$ using a long-baseline $\nu_{\mu} \rightarrow \nu_{e}$ appearance measurement, this ambiguity presents a problem since the oscillation probability is proportional to $\sin ^{2} \theta_{23}$ as shown in Equation 1. The present Super-K measurement would correspond to a change in the $\mathrm{T} 2 \mathrm{~K}$ or Nova oscillation probability of $70 \%$ for change in $\theta_{23}$ from $38^{0}$ to $52^{0}$.

This ambiguity in the determination of $\theta_{23}$ is difficult for a long-baseline $\nu_{\mu} \rightarrow \nu_{e}$ appearance measurement to resolve, but can be well addressed with combinations including reactor measurements. Figure 12 shows examples of different combinations of long-baseline results with (blue regions) or without (green regions) the inclusion of a medium scale reactor measurement. (The dashed red curve is for the inclusion of a small, Double-CHOOZ type measurement.) For this analysis, $\sin ^{2} 2 \theta_{23}=0.94 \pm 0.06, \sin ^{2} 2 \theta_{13}=0.05$, and $\delta_{C P}=270^{0}$. (Similar results are obtained for $\delta_{C P}=270^{\circ}$ ). As seen, the medium scale reactor data resolves this degeneracy when combined with any of the scenarios that include both neutrino and antineutrino data.

Future measurements of $\nu_{\mu}$ disappearance in the atmospheric $\Delta m^{2}$ region by the T2K or Nova experiments could reduce the uncertainty in $\sin ^{2} 2 \theta_{23}$ to of order 0.01 to 0.02 , but still leaving the $\theta_{23}$ vs. $\frac{\pi}{2}-\theta_{23}$ ambiguity. Figure 13 again shows the different combinations of long-baseline results with or without the inclusion of a medium scale reactor measurement. For this study, the future measurement is assumed with $\sin ^{2} 2 \theta_{23}=0.94 \pm 0.015$. Again, the medium scale reactor fully resolves the degeneracy when combined with any of the scenarios that include both neutrino and antineutrino data.

\subsection{Constraining CP Violation Parameters}

One of the important goals of an oscillation physics program is to determine if CP violating effects are present in the lepton sector as probed through the neutrino mixing matrix. In contrast to the reactor disappearance probability, the oscillation probabilities for the longbaseline experiments are effected by the value of the CP violation phase $\delta_{C P}$. Due to these 

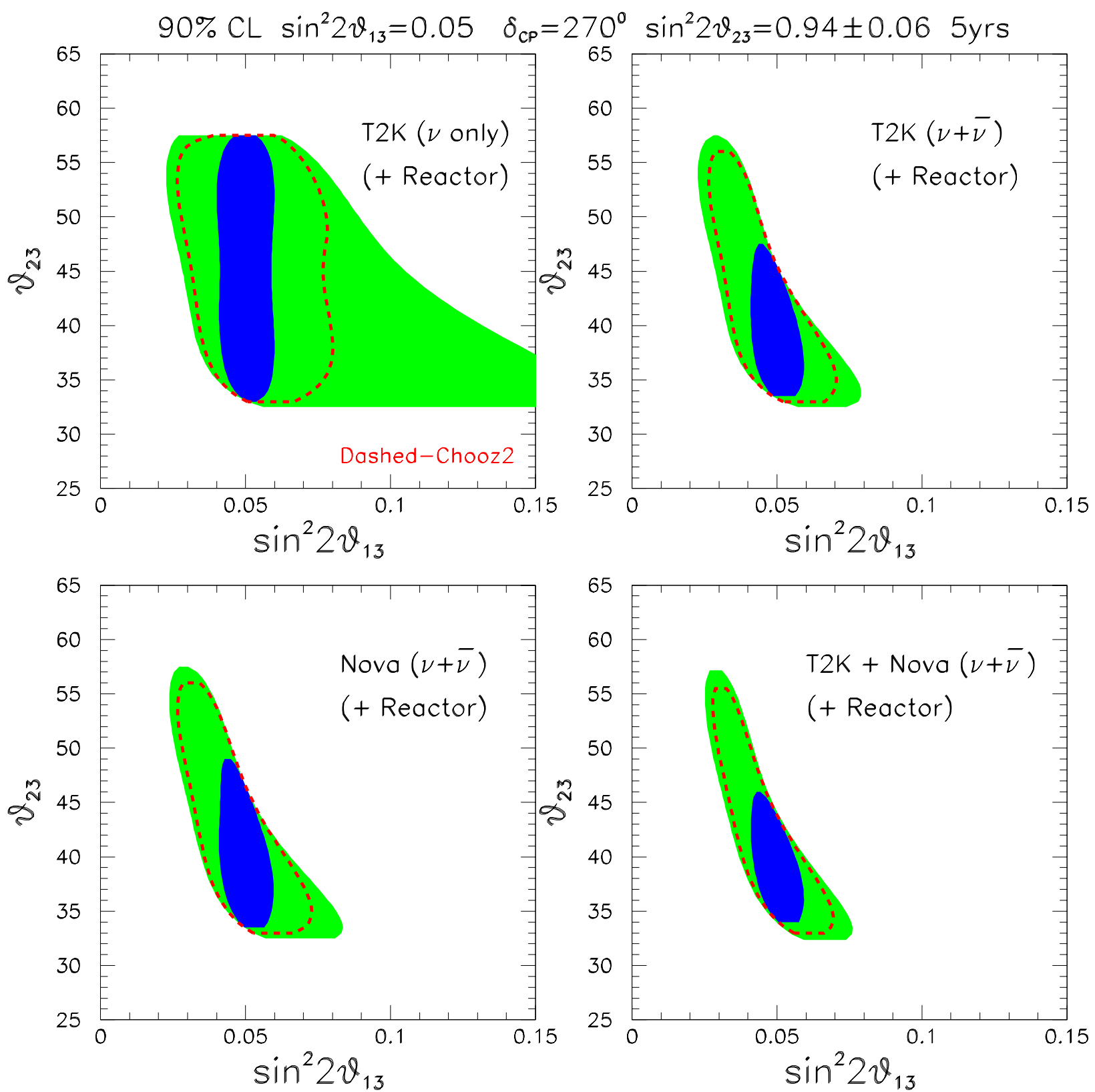

Figure 12: $90 \%$ C.L. allowed regions for simulated data with an underlying oscillation parameters of $\sin ^{2} 2 \theta_{13}=0.05, \theta_{23}=38^{0}, \Delta m^{2}=2.5 \times 10^{-3} \mathrm{eV}^{2}$ and $\delta_{C P}=270$. The analysis includes the restriction that $\sin ^{2} 2 \theta_{23}=0.94 \pm 0.06$. The green regions are for various long-baseline combinations of the T2K and/or Nova experiments for five years of running periods. The blue regions are the $90 \%$ C.L. allowed regions for a combined medium reactor plus long-baseline analysis. The dashed red lines indicate how the combined measurement would degrade with the small reactor sensitivity. 

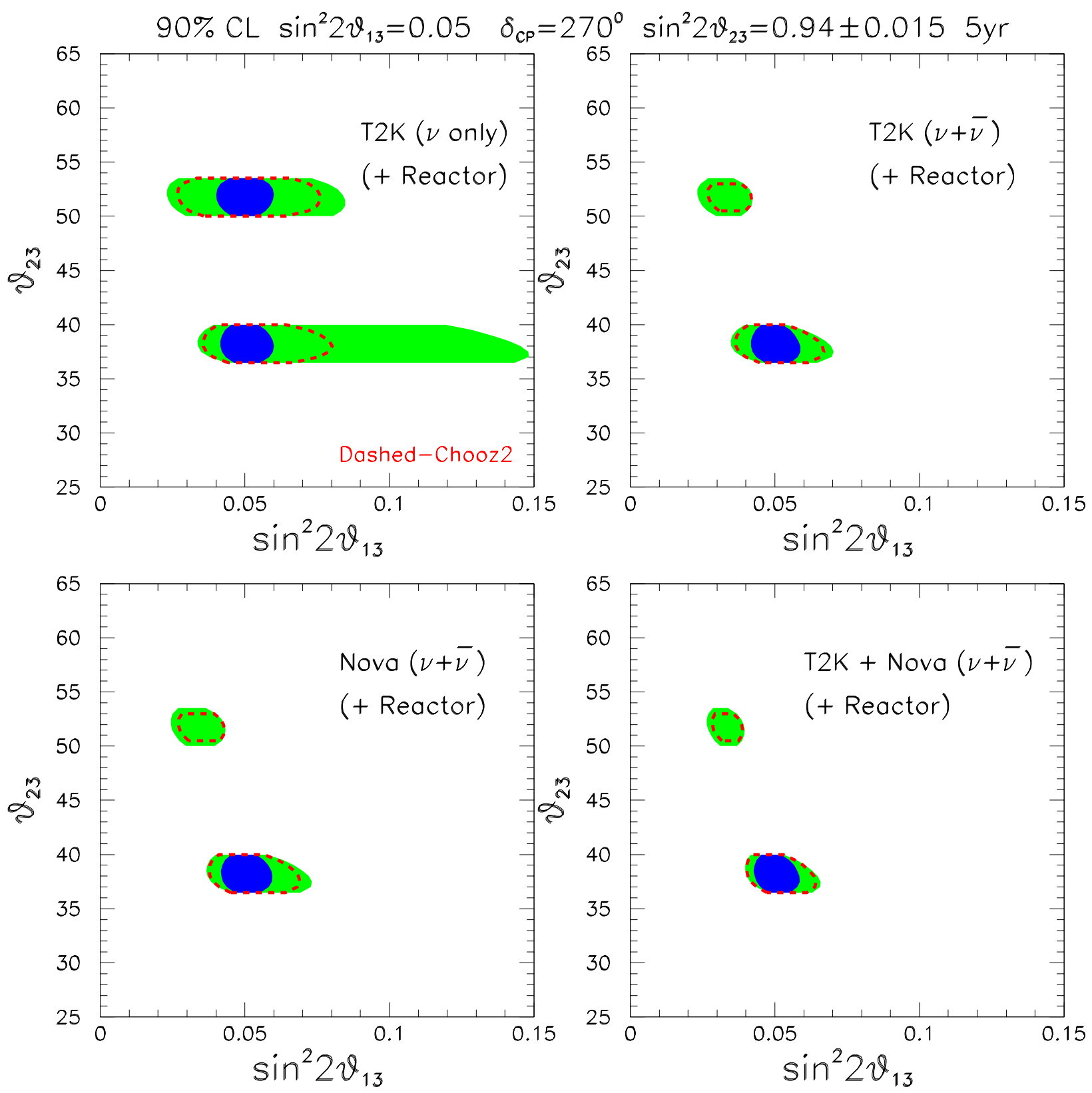

Figure 13: 90\% C.L. allowed regions for simulated data with an underlying oscillation parameters of $\sin ^{2} 2 \theta_{13}=0.05, \theta_{23}=38^{0}, \Delta m^{2}=2.5 \times 10^{-3} \mathrm{eV}^{2}$ and $\delta_{C P}=270$. The analysis includes the restriction that $\sin ^{2} 2 \theta_{23}=0.94 \pm 0.015$. The green regions are for various long-baseline combinations of the T2K and/or Nova experiments for five years of running periods. The blue regions are the $90 \%$ C.L. allowed regions for a combined medium reactor plus long-baseline analysis. The dashed red lines indicate how the combined measurement would degrade with the small reactor sensitivity. 
different types of behavior, combinations of long-baseline neutrino, anti-neutrino, and reactor measurements can be used to isolate these $\mathrm{CP}$ violating effects and place constraints on $\delta_{C P}$. The size of these effects are scaled by the value of $\sin ^{2} 2 \theta_{13}$ which is therefore an important parameter for setting the sensitivity to $\mathrm{CP}$ violation. The analysis also needs to include the uncertainties associated with the other parameters and especially the mass hierarchy. Figure 14 gives the $\nu_{\mu} \rightarrow \nu_{e}$ appearance oscillation probability as a function of $\delta_{C P}$ for the various combinations of beam type and mass hierarchy for $\sin ^{2} 2 \theta_{13}=0.06$.

¿From these figures, it can be seen that a measurement of the appearance probability for neutrino running alone could give information on $\delta_{C P}$ if the value of $\sin ^{2} 2 \theta_{13}$ was known for example from a reactor oscillation measurement. Figure 15 shows that the combinations of various measurements can be used to constrain the allowed CP violation phase. The results are for a scenario with $\sin ^{2} 2 \theta_{13}=0.05$ and the optimum phase point $\delta_{C P}=270^{\circ}$. In the top plot, a T2K $\nu$-only (5 years) measurement is displayed first without any reactor measurement (green region) then combined with a medium scale reactor measurement (blue region). (The dashed red curve outlines the region using a small scale (Double-CHOOZ type) reactor result.) The middle then shows what happens when both neutrinos (5 years) and anti-neutrinos (5 years) are used with and without the reactor measurement. Finally, the bottom plot shows the combination of T2K and Nova with and without the reactor result. The reactor measurement allows an early investigation of $\mathrm{CP}$ violation with a $\nu$-only long-baseline measurement and in all cases significantly reduces the uncertainty on $\theta_{13}$.

As a measure of how well the CP phase can be constrained in general, Figure 16 gives the regions in the $\delta_{C P}-\sin ^{2} 2 \theta_{13}$ plane for which a null $\mathrm{CP}$ violation solution is ruled out by at least two standard deviations. The dashed curves use long-baseline data only and the solid curves add data from a medium scale reactor experiment. The upper panels are for 5 years of running with neutrinos and antineutrinos assuming the rates shown in Table 6. Plot a) is for Nova only and b) combines data from both T2K and Nova. For the lower panels, it is assumed that there is a five-fold increase in intensity due, for example, to adding a Proton Driver at Fermilab. Plot c) is for Nova and d) combines Nova and T2K. From the plots, it is seen that the combination of T2K and Nova can probe the $\mathrm{CP}$ violation phase space if $\sin ^{2} 2 \theta_{13} \gtrsim 0.05(0.02)$ for nominal $(\times 5)$ beam rates. One also sees that the reactor experiment is important for resolving the degeneracy at $\delta_{C P}=180^{\circ}$. Also, shown in Figure 16 as vertical, red dashed-lines are the $90 \%$ CL upper limits for a small, medium, or large reactor experiment assuming a null underlying oscillation scenario. As seen, the reactor measurements will show how viable a $\mathrm{CP}$ violation measurement will be with the various combinations of the long-baseline setups. 

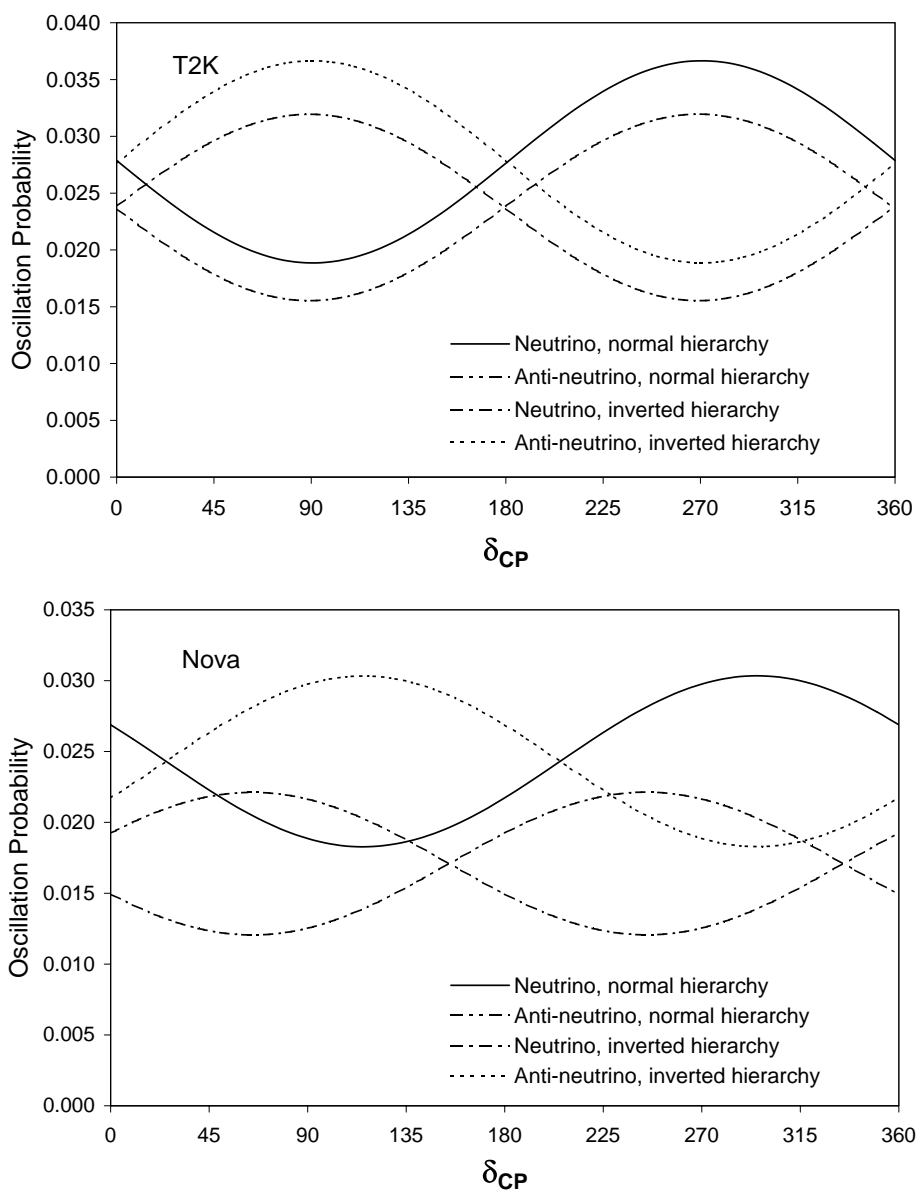

Figure 14: Oscillation probability for $\nu_{\mu} \rightarrow \nu_{e}$ appearance vs. $\delta_{C P}$ for the T2K (top) and Nova (bottom) experimental setups with $\Delta m^{2}=2.5 \times 10^{-3}$ and $\sin ^{2} 2 \theta_{13}=0.05$. The four curves correspond to pure neutrino or anti-neutrino beams with a normal or inverted hierarchy. 

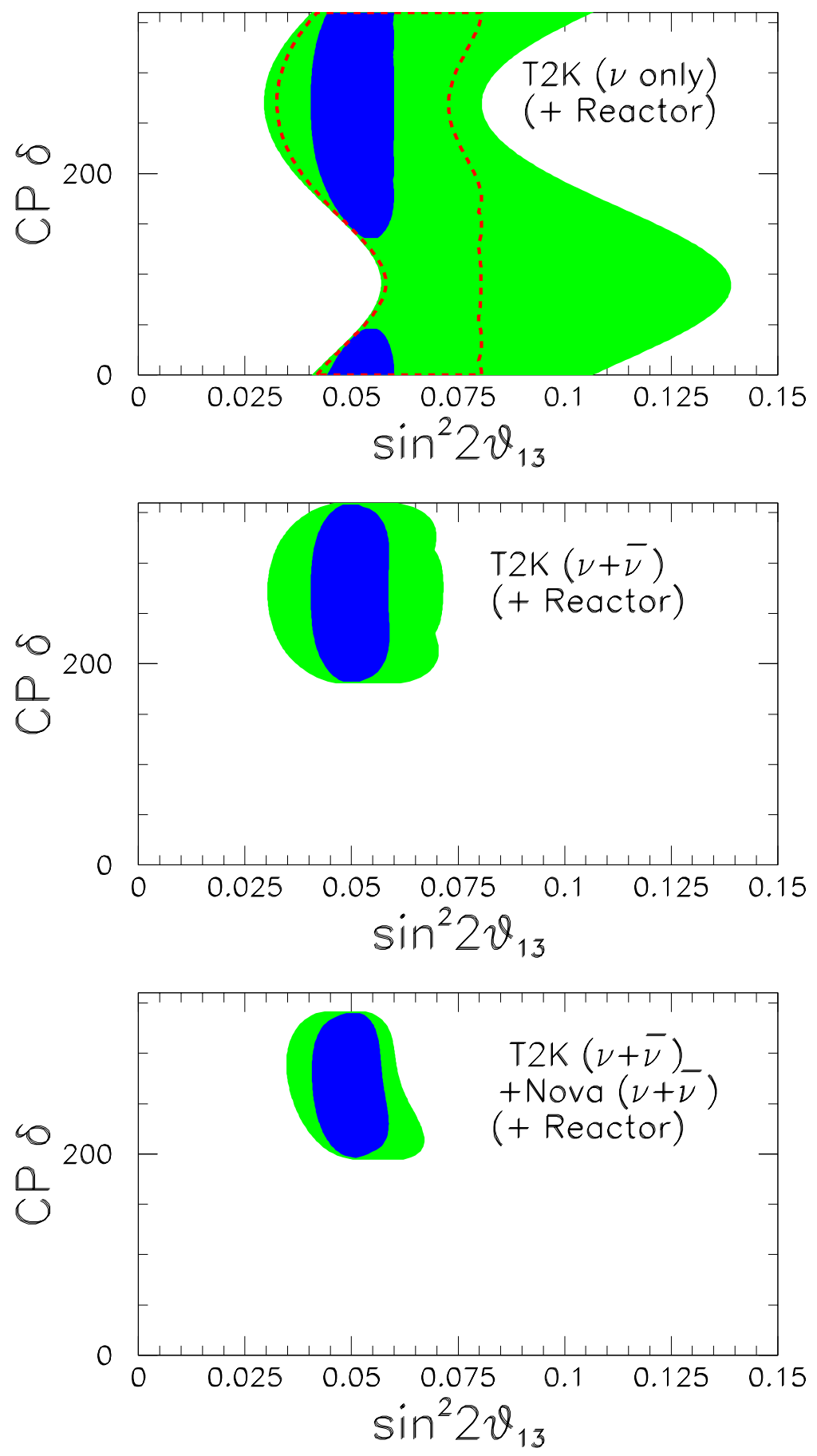

Figure 15: 90\% C.L. regions for various combinations of oscillation results for $\sin ^{2} 2 \theta_{13}=0.05$ and $\delta_{C P}=270^{\circ}$. Top: 5 year $\nu$-only T2K data with/without medium scale reactor results. Middle: $\mathrm{T} 2 \mathrm{~K} \nu+\bar{\nu}$ with/without reactor result. Bottom: $\mathrm{T} 2 \mathrm{~K} \nu+\bar{\nu}$ plus Nova $\nu+\bar{\nu}$ with/without reactor result. 

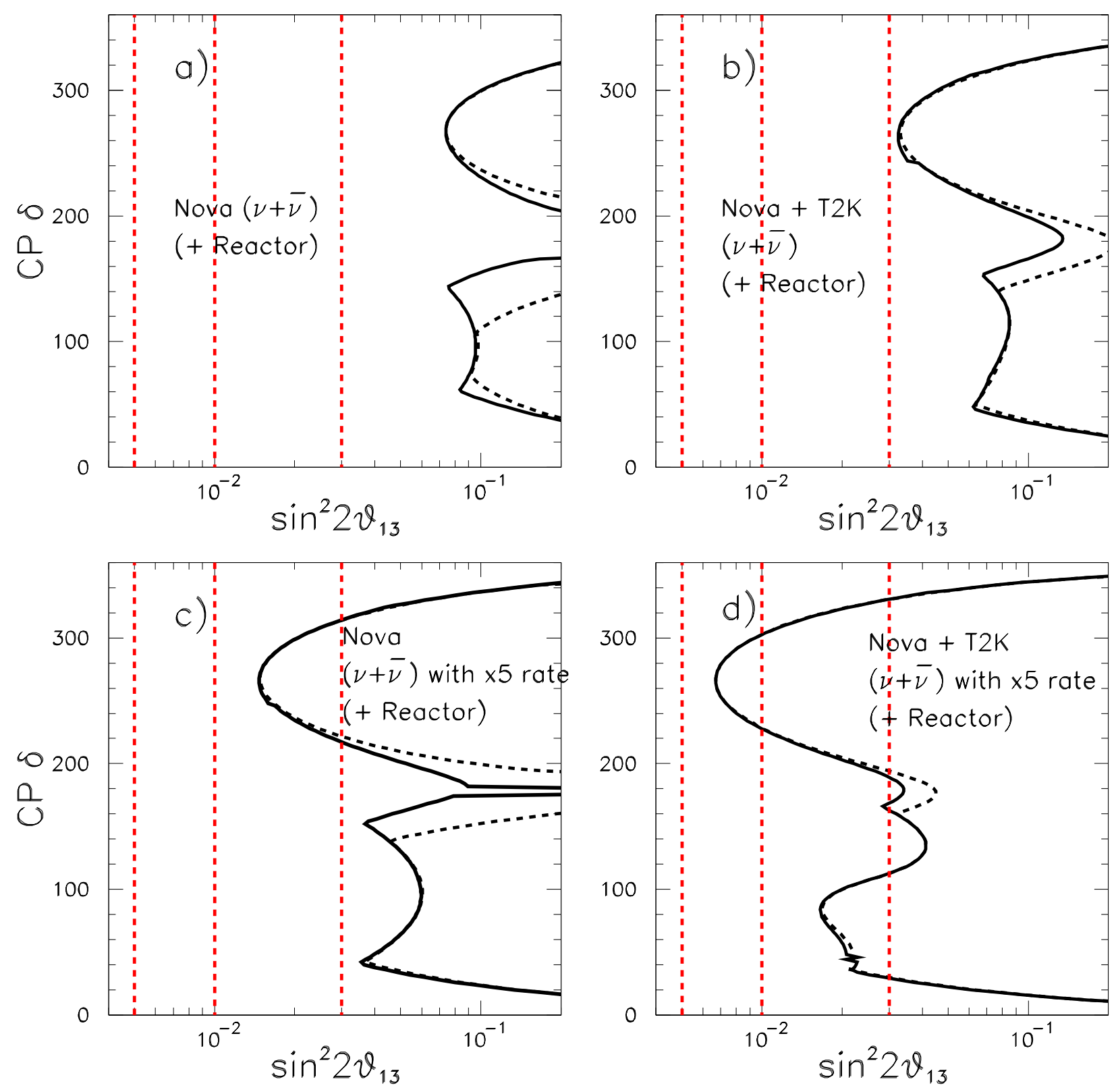

Figure 16: Regions in the $\delta_{C P}-\sin ^{2} 2 \theta_{13}$ plane for which a null CP violation solution is ruled out by at least two standard deviations. The dashed curves use long-baseline data only and the solid curves add data from a medium scale reactor experiment. a) Nova $\nu(5 \mathrm{yr})+\bar{\nu}(5 \mathrm{yr})$ data; b) T2K $\nu(5 \mathrm{yr})+\bar{\nu}(5 \mathrm{yr})+$ Nova $\nu(5 \mathrm{yr})+\bar{\nu}(5 \mathrm{yr})$ data; $)$ Nova $(\times 5$ rate with Proton Driver $) \nu(5 \mathrm{yr})+\bar{\nu}(5 \mathrm{yr})$ data; $\mathrm{d}) \mathrm{T} 2 \mathrm{~K}(\times 5$ rate $) \nu(5 \mathrm{yr})+\bar{\nu}(5 \mathrm{yr})+$ Nova $(\times 5$ rate $) \nu(5 \mathrm{yr})+$ $\bar{\nu}(5 \mathrm{yr})$ data. The vertical dashed lines give the $90 \%$ CL upper limit sensitivity for a small, medium, and large reactor experiment. 


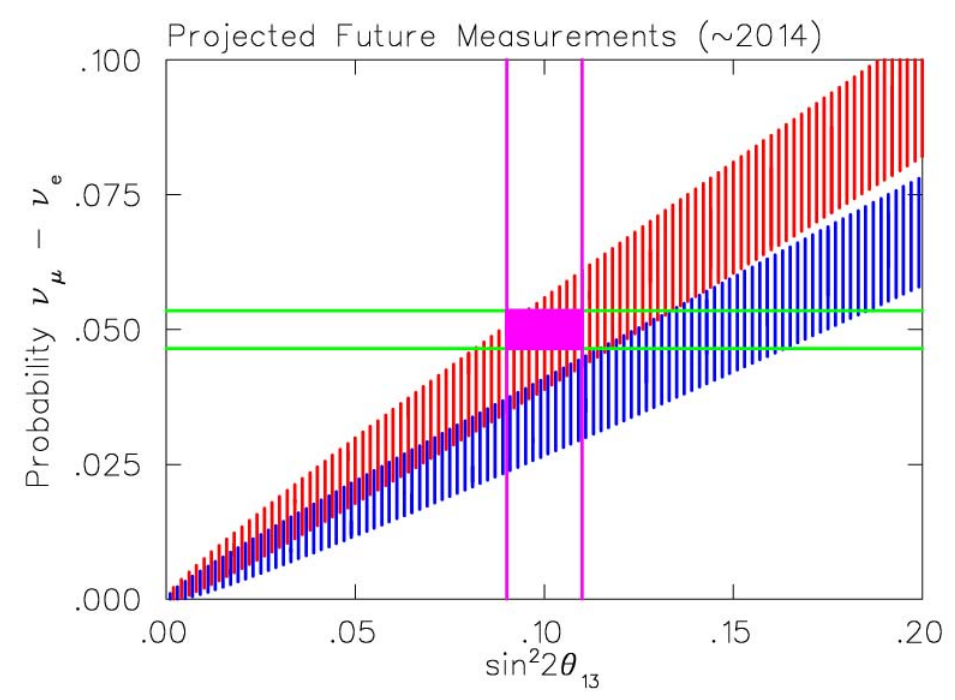

Figure 17: Example of how a medium scale reactor measurement and a 5 year $\nu$-only Nova measurement can constrain the mass hierarchy.

\subsection{Determining the Mass Hierarchy}

For constraining the mass hierarchy, one needs to compare measurements in a region where the oscillation probability changes significantly for a normal versus inverted mass spectrum (See Figure 14). The Nova experiment is particularly important here due to their long pathlength in matter. Figure 17 shows the Nova $\nu_{\mu} \rightarrow \nu_{e}$ oscillation probability versus $\sin ^{2} 2 \theta_{13}$. The two sets of vertical bars show the variation with $\delta_{C P}$ for the normal (top) and inverted (bottom) hierarchy. A medium scale reactor experiment will constrain the value of the $\sin ^{2} 2 \theta_{13}$ to the region delineated by the vertical lines. Combining this with a 5 year Nova measurement of the oscillation probability could, if the parameters are favorable, determine which hierarchy was allowed.

A more accurate determination of the hierarchy is possible by combining the results from long-baseline neutrino and antineutrino data. Here again the ambiguity with respect to the value of $\delta_{C P}$ limits the determination to regions in the $\sin ^{2} 2 \theta_{13}-\delta_{C P}$ plane. Figure 18 shows the regions for which the mass hierarchy is resolved by two standard deviations. The dashed curves use long-baseline data only and the solid curves add data from a medium (or large) scale reactor experiment. The various panels in the figure are for data sets given by: a) Nova for $\nu(5 \mathrm{yr})$ only data and $\nu(3 \mathrm{yr})$ plus $\bar{\nu}(3 \mathrm{yr})$ data; b) Nova plus T2K for $\nu(3 \mathrm{yr})$ plus $\bar{\nu}(3 \mathrm{yr})$ data; c) Nova with $\times 5$ the nominal beam rate for $\nu(3 y r)$ plus $\bar{\nu}(3 \mathrm{yr})$ data; d) T2K and Nova with $\times 5$ the nominal beam rate, each for $\nu(3 \mathrm{yr})$ plus $\bar{\nu}(3 \mathrm{yr})$. 
The plots show that the mass hierarchy can be determined for limited regions with $\sin ^{2} \theta_{13}>0.05$ for the nominal beam rates and $>0.025$ for the enhanced $(\times 5)$ rates. The addition of the $\mathrm{T} 2 \mathrm{~K}$ and reactor data helps improve the measurement reach for the bad $\delta_{C P}$ regions.

\subsection{Conclusions}

Reactor measurements hold the promise of constraining or measuring the $\theta_{13}$ mixing parameter and resolving the ambiguity in determining the $\theta_{23}$ mixing parameter. The size of these parameters are important inputs for models of lepton mass and mixing that span the range from GUTs trying to relate the CKM and MNS matrix to extra dimension models that have neutrinos propogating in the bulk. The smallness of $\theta_{13}$ relative to the other anlges may be giving us a hint as to what the underlying theory may be. Besides leading to a better understanding of neutrino mixing, these angles, $\theta_{13}$ and $\theta_{23}$, are two of the twenty six parameters of the standard model and, as such, are worthy of high precision measurement independently of other considerations. For $\theta_{13}$, a two-detector reactor experiment unambiguously measures the size of this angle with significantly better precision than any other proposed experimental technique and reactor data may be key for determining $\theta_{23}$.

The size of $\theta_{13}$ is also important for interpreting long-baseline experiments and for planning a viable future program neutrino oscillations program. In addition to setting the scale for future studies, a reactor result when combined with long-baseline measurements may also give early constraints on $\mathrm{CP}$ violation and early indications of the mass hierarchy. The combination of the $\mathrm{T} 2 \mathrm{~K}$ and Nova long-baseline experiments will be able to make significant measurements of these effects if $\sin ^{2} 2 \theta_{13}>0.05$ and with enhanced beam rates can improve their reach to the $\sin ^{2} 2 \theta_{13}>0.02$ level. If $\theta_{13}$, as measured by a reactor experiment, turns out to be smaller than these values, one will need other strategies for getting to the physics. Thus, an unambiguous reactor measurement of $\theta_{13}$ is an important ingredient in planning the strategy for this program as well as accessing the phenomenology of neutrino mixing.

\section{Beyond $\theta_{13}$}

In this section, we review other physics possibilities using anti-electron neutrinos from a nuclear reactor. These include a measurement of the weak mixing angle, improved limits on neutrino magnetic moments, an improved measurement of $\Delta m_{12}^{2}$, tests of CPT symmetry, searches for sterile neutrinos, and studies of nearby supernova. 

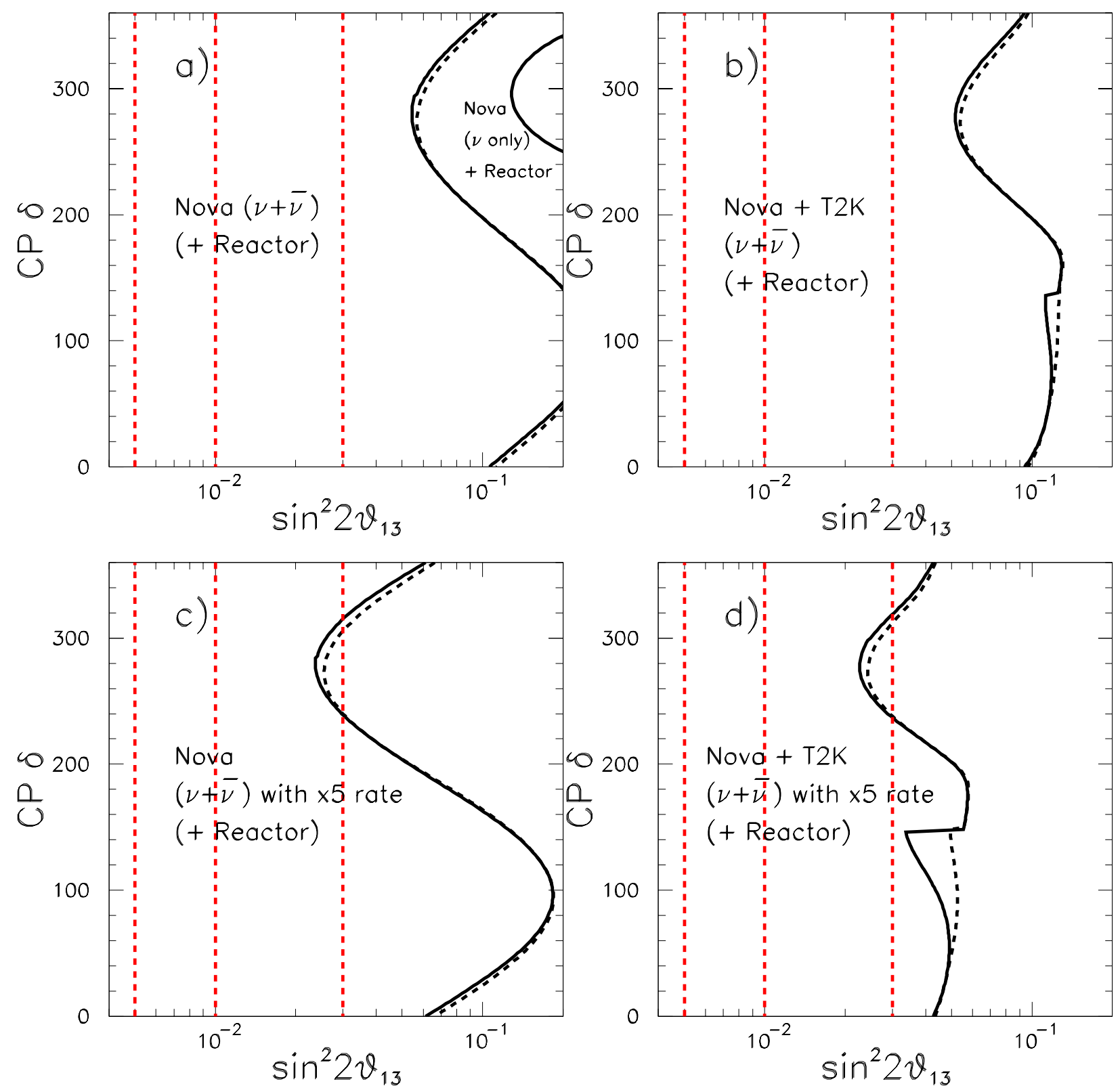

Figure 18: Regions in the $\delta_{C P}-\sin ^{2} 2 \theta_{13}$ plane for which the mass hierarchy is resolved by two standard deviations. The dashed curves use long-baseline data only and the solid curves add data from a medium scale reactor experiment. a) Nova $\nu(5 \mathrm{yr})$ only data and $\nu(3 \mathrm{yr})+$ $\bar{\nu}(3 \mathrm{yr})$ data; b) Nova plus T2K with $\nu(3 \mathrm{yr})+\bar{\nu}(3 \mathrm{yr})$ data; $\mathrm{c})$ Nova $(\times 5$ beam rate $)$ with $\nu(3 \mathrm{yr})+\bar{\nu}(3 \mathrm{yr})$ data; $\mathrm{d}) \mathrm{T} 2 \mathrm{~K}(\times 5$ beam rate rate $) \nu(3 \mathrm{yr})+\bar{\nu}(3 \mathrm{yr})+$ Nova $(\times 5$ beam rate $)$ $\nu(3 \mathrm{yr})+\bar{\nu}(3 \mathrm{yr})$ data. 


\section{1 $\sin ^{2} \theta_{W}$}

Anti-neutrino-electron elastic scattering, $\bar{\nu}_{e}+e^{-} \rightarrow \bar{\nu}_{e}+e^{-}$, at low momentum provides unique access to the electroweak theory. First, the scattering involves only leptons. Second, a precision measurement may be made at very low momentum transfer. Finally, the process involves both neutral and charged weak currents. These three features make a compelling case for carrying out a measurement of the $\bar{\nu}_{e} e^{-}$cross section using reactor neutrinos.

Measurements carried out by $\mathrm{NuTeV}$ stand three standard deviations from predictions based on data from LEP I, SLC, CDF and D0, Fig. 19 and a precision measurement of $\bar{\nu}_{e} e^{-}$scattering would play a major role in indicating the nature of this discrepancy. For $\bar{\nu}_{e} e^{-}$scattering at a reactor, $Q^{2}<m_{\pi}^{2}$, which means hadronic corrections play a much smaller role than measurements at higher momentum transfer. Since in $\bar{\nu}_{e} e^{-}$scattering involves only leptons, comparison of a $\bar{\nu}_{e} e^{-}$measurement with $\mathrm{NuTeV}$ distinguishes between leptonic and hadronic neutral current couplings. Finally, the presence of both charged and neutral currents lifts the degeneracy between $G_{F}$ and $\rho$ which occurs in processes which only involve neutral currents.

Ref. [14] gives a model in which separating contributions from $G_{F}$ and $\rho$ is important: the model explains the $\mathrm{NuTeV}$ result by reducing the neutrino coupling and keeping $\rho$ close to one to preserve the $Z$ pole measurements. The reduction in the neutrino coupling by a factor of $\epsilon=0.003 \pm 0.001$ (explained by mixing a heavy sterile neutrino with the light neutrinos) explains the $\mathrm{NuTeV}$ result. A measurement of $\bar{\nu}_{e} e^{-}$scattering with a precision of $1.3 \%$ would be sensitive to changes in he neutrino couplings at this level.

Even in the absence of a discrepancy, a precision measurement of $\bar{\nu}_{e} e^{-}$using reactor neutrinos will be important [16]. Reaching a precision below $1 \%$ provides access contact interactions (via $Z^{\prime}$ exchange), neutrino magnetic moments, dynamical symmetry breaking and matter oscillations in high densities. More broadly, the unique nature of $\bar{\nu}_{e} e^{-}$make their measurement important independently of other considerations; it is a fundamental process and hence always worthy of study.

We aim to measure the $\bar{\nu}_{e} e^{-}$cross section with a accuracy of around $1 \%$, which requires a minimum of 10,000 events and the control of systematics at a comparable level. The near detectors for the $\theta_{13}$ measurement discussed above will provide ample statistics in three years running. For the systematic errors, two problems immediately present themselves: the determination of the incident anti-neutrino flux and the reduction of backgrounds.

Starting from a known fuel composition, the anti-neutrino flux from a reactor may be calculated with an accuracy of $2 \%$. Since the relative error in the spectrum increases with anti-neutrino energy, fitting the shape of the recoil electron spectrum may reduce the error. However, a more elegant solution presents itself [17]: we normalize the $\bar{\nu}_{e} e^{-}$rate using the neutrino absorption $\bar{\nu}_{e}+p \rightarrow e^{+}+n$ rate to determine the anti-neutrino flux. For 


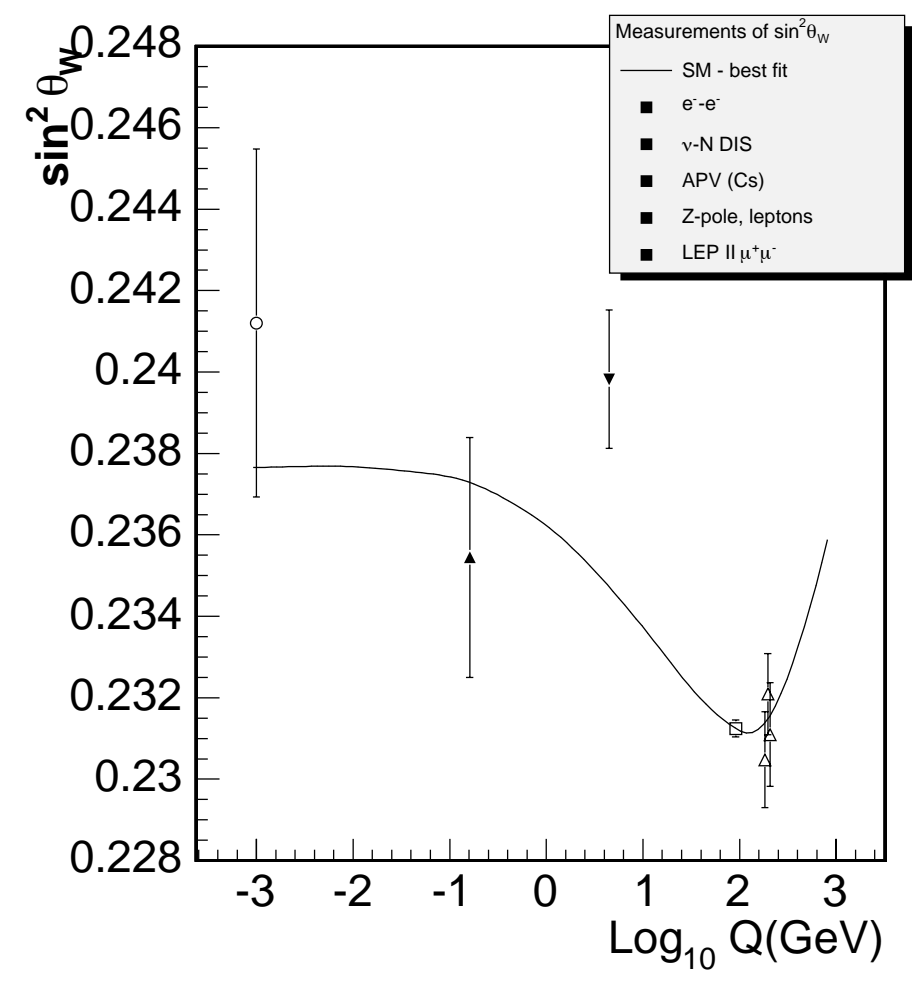

Figure 19: Measurements of $\sin ^{2} \theta_{W}$ as a function of $Q$; The combined measurements of LEP I, CDF, D0 and SLC determine the solid curve. The other experimental measurements are shown for comparison. Not all measurements are shown.

neutrino absorption, $E_{e^{+}}=E_{\bar{\nu}}-\left(M_{n}-M_{p}\right)-m_{e}$, so a measurement of the positron energy spectrum gives a measurement of the anti-neutrino energy spectrum which may be applied to extract the $\bar{\nu}_{e} e^{-}$cross section from the measured number of events. The measurement of $\theta_{13}$ described above requires knowledge of the detector efficiency to a higher precision than required for the flux measurement.

Reduction of backgrounds presents a more complex problem. We plan to carry of the measurement of the $\bar{\nu}_{e} e^{-}$cross section in the energy range $3 \leq E_{v i s} \leq 5 \mathrm{MeV}$, where $E_{v i s}$ is the visible energy. This is a region between two major backgrounds: radioactive contaminants below $3 \mathrm{MeV}$ and unassociated neutrons above $5 \mathrm{MeV}$. Both sources may be mitigated somewhat by locating the detectors at 300 MWE or deeper, but a great deal of care must be taken to reduce backgrounds from radio-contaminants and neutrons.

A detailed study [17] shows a precision of $1 \%$ or better may be achieved in three years running at the Braidwood site with minor modification to the detectors. First, as neutrino absorption constitutes an important background for $\bar{\nu}_{e} e^{-}$if the neutron is not detected, 
the $\bar{\nu}_{e} e^{-}$measurement requires efficient detection of neutron capture on hydrogen. Second, reduction of background from muon induced isotopes requires a large overburden, $300 \mathrm{MWE}$ at least. A larger overburden would provide greater reduction, but must be balanced against the loss of rate from the reduction in solid angle. Finally, we require purification of the gadolinium and oil at the level of KamLAND; the low energy cut of $3 \mathrm{MeV}$ eliminates the single photon and alpha backgrounds, leaving only the correlated $\beta-\gamma$ decays which can only be reduced by careful control of contaminants.

Given the importance of measuring $\bar{\nu}_{e} e^{-}$precisely, one could argue for a dedicated experiment. However, being part of a system of detectors at different baselines to measure $\theta_{13}$ make the measurement of environmental background rates in situ. The measurement of muon induced events in the far detector determines the rate of muon induced events in the near detector allowing their subtraction from the $\bar{\nu}_{e} e^{-}$window in the near detector. Having the detectors as identical as possible makes the subtraction simpler. We assume that the Gd-doped scintillator is mixed in a single batch so that contaminants are the same in all detectors. The overburdens may not be identical, but in the flat environment of Braidwood they will be very similar. Table 7 summarizes systematic errors.

Lorentz invariance requires Majorana neutrinos have no magnetic moment. For Dirac neutrinos, the electroweak theory predicts a magnetic moment of $\nu_{\nu}=10^{-19} \nu_{B} /<m_{\nu_{e}}>$ where $\left\langle m_{\nu_{e}}\right\rangle$ is the mixing weighted mass. For a model with the three known light neutrinos, the predicted magnetic moment of $<10^{-19} \mu_{B}$ presents an insurmountable experimental challenge. However, mixing with heavy states [16] or coupling with through extra dimensions [18] could lead to measurable magnetic moment effects.

A neutrino magnetic moment increases the $\bar{\nu}_{e} e^{-}$cross section at low recoil energies by a factor of $\left(1 / T-1 / E_{\nu}\right)$, where $T$ denots the electron recoil kinetic energy. Measuring the shape of the electron recoil spectrum for $\bar{\nu}_{e} e^{-}$events separates the magnetic moment contribution from the weak interaction contribution allowing the measurement of the magnetic moment. Our studies show a measurement of the $\bar{\nu}_{e} e^{-}$cross section with $1 \%$ detection gives sensitivity to $3 \times 10^{-11} \mu_{B}$ which would improve on the current limits of $10^{-10} \mu_{B}$ [19].

In summary, the measurement of $\bar{\nu}_{e} e^{-}$using reactor neutrinos with a precision around $1 \%$ is very important scientifically and possible technically with little modification to the apparatus we propose to measure $\theta_{13}$. 
Table 7: Fractional errors on the number of $\bar{\nu} e$ scattering events based on assumptions presented in ref. [17].

\begin{tabular}{|c|c|}
\hline Statistical error on the signal & $0.95 \%$ \\
Statistical error $\bar{\nu} p$ background subtraction & $0.34 \%$ \\
Statistical error on U and Th background & $0.09 \%$ \\
Statistical error on muon-induced isotopes & $0.34 \%$ \\
Systematic error on muon-induced isotopes & $0.46 \%$ \\
Statistical error on the normalization & $0.10 \%$ \\
Systematic error on electron-to-free-proton ratio & $0.60 \%$ \\
Systematic error on the Gd capture fraction & $0.30 \%$ \\
\hline Total error & $1.3 \%$ \\
\hline
\end{tabular}

\subsection{Precision Measurement of $\theta_{12}$ with a Second Generation Long Baseline Reactor Experiment}

\subsubsection{Physics Motivation}

High precision measurementd of the $(1,2)$ sector mixing parameters $-\Delta m_{21}^{2}$ and $\sin ^{2} \theta_{12}$, which drive the solar neutrino oscillations, are of fundamental importance from the standpoint of neutrino physics and solar astrophysics. The two parameters $\Delta m_{21}^{2}$ and $\sin ^{2} \theta_{12}$ belong to the set of basic parameters characterizing 3-neutrino mixing and oscillations. The knowledge of the precise values of $\Delta m_{21}^{2}$ and $\sin ^{2} \theta_{12}$ is first of all of crucial importance for understanding the origin of neutrino masses and mixing. The comparison of the results of reactor $\bar{\nu}_{e}$ experiments with those obtained in current and future solar neutrino experiments provides a unique test of the neutrino oscillation theory and its quantum mechanics foundations. The reactor and solar neutrino experiments differ in almost every relevant way: the baselines are vastly different, the lepton charges carried by $\nu_{e}$ and $\bar{\nu}_{e}$ are opposite, the matter (MSW) effects play important role in the solar $\nu_{e}$ transitions and are unimportant for the reactor $\bar{\nu}_{e}$ oscillations.

In addition to tests of neutrino physics, reactor measurements of $\Delta m_{21}^{2}$ and $\sin ^{2} \theta_{12}$ provide strong constraints on solar physics. Independent knowledge of these parameters allows us to predict the total fluxes of the low energy solar $p p$ neutrinos using exclusive measurements of the solar $\nu_{e}$ flux (and in the absence of inclusive neutral current measurements of the flux) [20]. For the higher energy solar $\left({ }^{8} \mathrm{~B}\right)$ neutrinos, a comparison of the predicted total flux, based upon measurements (on Earth) of the $\nu_{e}$ component of the flux and on reactor-based determination of $\Delta m_{21}^{2}$ and $\sin ^{2} \theta_{12}$, and of the measured total active neutrino flux through neutral current reaction, allow tests for possible transitions of the solar $\nu_{e}$ into 
sterile neutrinos.

A measurement of $\bar{\nu}_{e}$ disapperance with smaller uncertainties in a KamLAND-like long baseline reactor experiment could also provide an indirect measure of $\theta_{13}$ when combined with solar neutrino measurements. Because the solar $\nu_{e}$ transitions change from matter-enhanced at high energies ( ${ }^{8} \mathrm{~B}$ neutrinos) to vacuum-dominated at low energies ( $p p$ neutrinos), the low and high energy solar neutrino data allows a measurement of $\theta_{12}$ which is only weakly correlated with $\theta_{13}$. This fact, in combination with a precision terrestrial measurement of

$\bar{\nu}_{e}$ disappearance driven by $\Delta m_{21}^{2}$ and $\sin ^{2} \theta_{12}$, like that made by KamLAND, can lead to a restriction on the size of $\theta_{13}$.

\subsubsection{Long Baseline Reactor Experiments}

Long baseline reactor $\bar{\nu}_{e}$ experiments have a remarkable potential for high precision determination of $\Delta m_{21}^{2}$ and $\sin ^{2} \theta_{12}$. In what regards the $\Delta m_{21}^{2}$, this was convincingly demonstrated by the KamLAND experiment. Under the plausible assumption of CPT-invariance, the first results from the KamLAND [21] experiment, when combined with the data from the solar neutrino experiments, not only have confirmed the existence of $\nu_{e} \rightarrow \nu_{\mu, \tau}$ oscillations of solar neutrinos, but have also constrained considerably the allowed values of $\Delta m_{21}^{2}$, establishing the large mixing angle (LMA) MSW solution essentially as the unique solution of the solar neutrino problem [21].

Recently the KamLAND collaboration reported new data [22] on the spectrum of $e^{+}$ produced by reactor $\bar{\nu}_{e}: \bar{\nu}_{e}+p \rightarrow e^{+}+n$. The data correspond to a statistics of 766.3 Ty and clearly show a distortion of the $e^{+}$-spectrum, compatible with that predicted in the case of $\bar{\nu}_{e}$ oscillations, driven by $\Delta m_{21}^{2}$ lying in the low-LMA subregion of the LMA solution region (see, e.g., [21]). The combined solar neutrino and KamLAND 766.3 Ty spectrum data permitted to determine $\Delta m_{21}^{2}$ with a much better precision than that reached before the latest KamLAND results were available. This is illustrated in Fig. 1 taken from [22], in which the allowed region of $\Delta m_{21}^{2}$ and $\tan ^{2} \theta_{12}$, obtained in a two-neutrino oscillation analysis of the solar and KamLAND 766.3 Ty data is shown. The allowed values of the two parameters are given by: $\Delta m_{21}^{2}=\left(8.2_{-0.5}^{+0.6}\right) \times 10^{-5} \mathrm{eV}^{2}, \tan ^{2} \theta_{12}=\left(0.40_{-0.07}^{+0.09}\right)$. The dependence on $\Delta m_{21}^{2}$ of the relevant $\chi^{2}$-function, shown in Figure 21 (right panel), demonstrates [23] that the KamLAND results are crucial for the quoted above high precision determination of $\Delta m_{21}^{2}$. Future KamLAND data can allow to determine $\Delta m_{21}^{2}$ with an uncertainty approximately of $10 \%$ at $99 \%$ C.L. $[24,25]$.

The KamLAND results, however, had so far little impact on the precision of measurement of the solar neutrino mixing angle $\theta_{12}$, which is determined essentially by the solar neutrino 


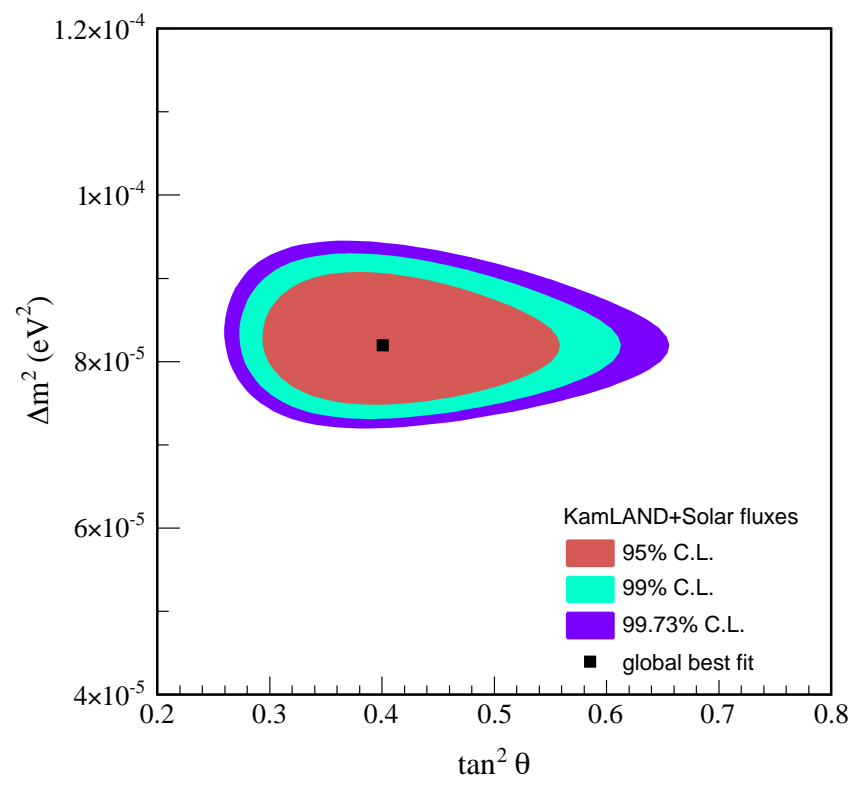

Figure 20: Result of a combined two-neutrino oscillation analysis of the KamLAND 766.3 Ty spectrum data and the global solar neutrino data performed under the assumption of CPT invariance (from ref. [22]).

data [26]. Figure 21, left panel, clearly illustrates this fact. As was discussed in detail in $[24,25]$, the uncertainty in the measured value of $\theta_{12}$ will not be reduced substantially with respect to the presently existing one quoted above (Fig. 1) even after high statistics data from KamLAND will be available, and even after the Shika-2 reactor complex, located at approximately $88 \mathrm{~km}$ from KamLAND, will become operational in 2006. For the current best fit value of $\Delta m_{21}^{2}$, the dominant part of the signal in the KamLAND experiment is due to $\bar{\nu}_{e}$ (essentially from the Kashiwazaki complex of reactors located at a distance $L$ of about $160 \mathrm{~km}$ ), for which $\sin ^{2}\left(\Delta m_{21}^{2} L /(4 E)\right) \cong 0$. This corresponds to a maximum of the $\bar{\nu}_{e}$ survival probability (SPMAX), $P\left(\bar{\nu}_{e} \rightarrow \bar{\nu}_{e}\right)=1-\sin ^{2} 2 \theta_{12} \sin ^{2}\left(\Delta m_{21}^{2} L /(4 E) \cong\right.$ 1 , and, correspondingly, the sensitivity to $\sin ^{2} 2 \theta_{12}$ is relatively low [26]. Although the Shika-2 reactors are located at a distance which, in principle, could allow a more precise measurement of $\sin ^{2} 2 \theta_{12}$, they are not powerful enough to improve significantly the sensivity of the KamLAND experiment to $\theta_{12}$ [24]. Future data from SNO [27] and KamLAND experiments can lead to a reduction of the uncertainty in the value of $\sin ^{2} \theta_{12}$ to $17 \%$ at $99 \%$ C.L. [25]. Data from the future solar neutrino experiments designed to measure the low energy $p p$ flux with a relative precision of $3 \%(1 \%)$ are expected to restrict the uncertainty in the measured values of $\sin ^{2} \theta_{12}$ to $18 \%(12 \%)$ at $99.73 \%$ C.L., while the data from the BOREXINO experiment is not expected to lead to any significant improvements in the precision of $\sin ^{2} \theta_{12}$ determination [20]. 


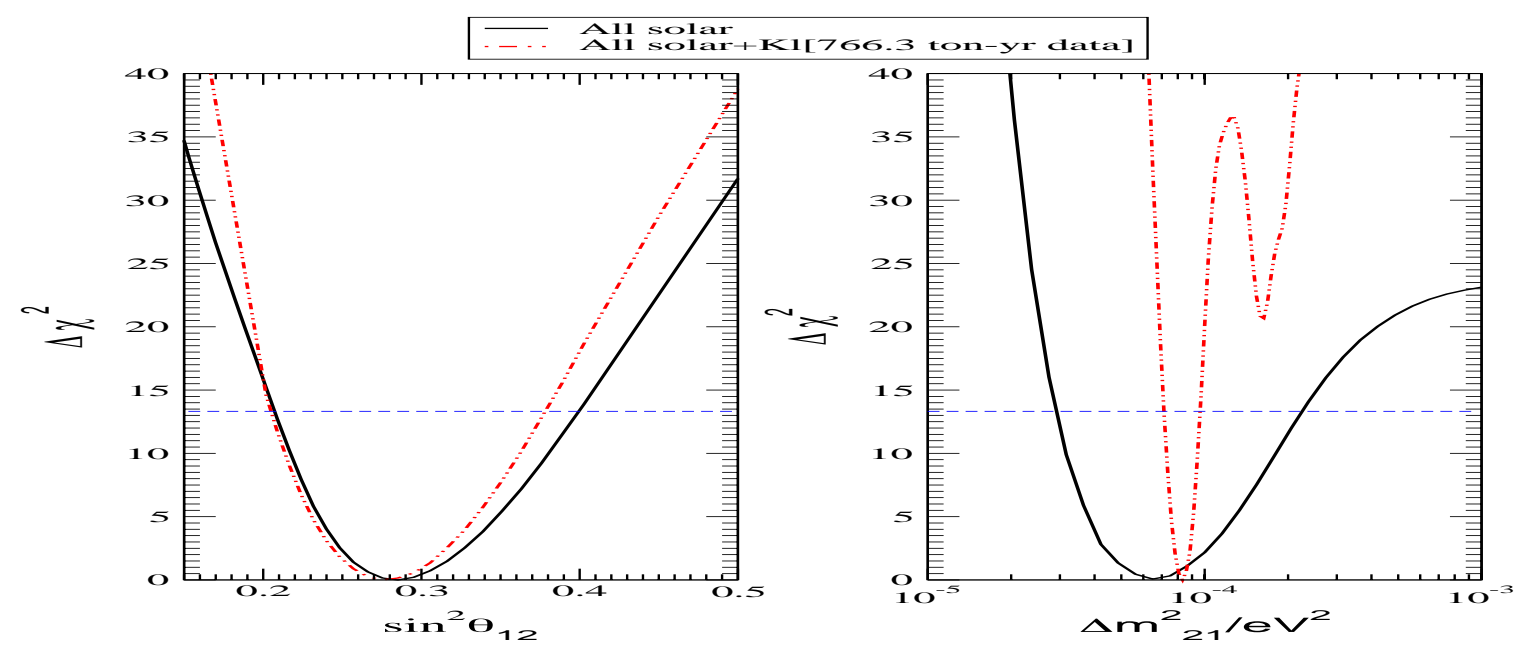

Figure 21: The dependence of the $\Delta \chi^{2}$-function in the analysis of the KamLAND 766.3 Ty spectrum and solar neutrino data on $\Delta m_{21}^{2}$ (right panel) and on $\sin ^{2} \theta_{12}$ (left panel). The results shown in both panels are obtained by allowing all the other parameters to vary freely. The dashed line shows the $3 \sigma$ limit corresponding to 1 parameter fit (from ref. [23]).

The highest precision in the measurement of $\theta_{12}$ can be achieved in a reactor experiment with a baseline for which the maximum of the event rate spectrum in the absence of $\bar{\nu}_{e}$ oscillations (located at $E \sim 3.6 \mathrm{MeV})$ corresponds to $\sin ^{2}\left(\Delta m_{21}^{2} L /(4 E)\right) \cong 1$, i.e., to a minimum of the $\bar{\nu}_{e}$ survival probability (SPMIN) [26]: $P\left(\bar{\nu}_{e} \rightarrow \bar{\nu}_{e}\right) \cong 1-\sin ^{2} 2 \theta_{21}$. For the current best fit value of $\Delta m_{21}^{2}$ such an experiment should have a baseline of $L \cong(55-60)$ $\mathrm{km}$ [23]. Thus, a high precision measurement of the mixing angle $\theta_{12}$ requires a second generation long baseline reactor experiment.

Note that the largest uncertainties in the KamLAND data so far have been the statistical one and the systematic uncertainty related to the lack of precise knowledge of the fiducial mass of the detector. In addition, there are also the systematic uncertainties associated with the uncertainties in the knowledge of the reactor $\bar{\nu}_{e}$ flux (roughly $2 \%$ ), spectrum (2.5\%) and $\bar{\nu}_{e}+p \rightarrow e^{+}+n$ cross section $(0.2 \%)$.

\subsubsection{Potential Experiment}

Since KamLAND is expected to measure $\Delta m_{21}^{2}$ with very high precision, we will focus on the measurement of $\theta_{12}$ in what follows. A new experiment aiming at high precision measurement of $\theta_{12}$ could have several advantages. A single baseline, rather than the multiple baselines relevant for KamLAND due to the multiple reactors contributing to the signal, could make a spectral distortion clearer. In addition, if a new $\Delta m_{21}^{2}$ and $\sin ^{2} \theta_{12}$ reactor experiment were to run in conjunction with a short baseline $\theta_{13}$ experiment, many of the uncertainties on 

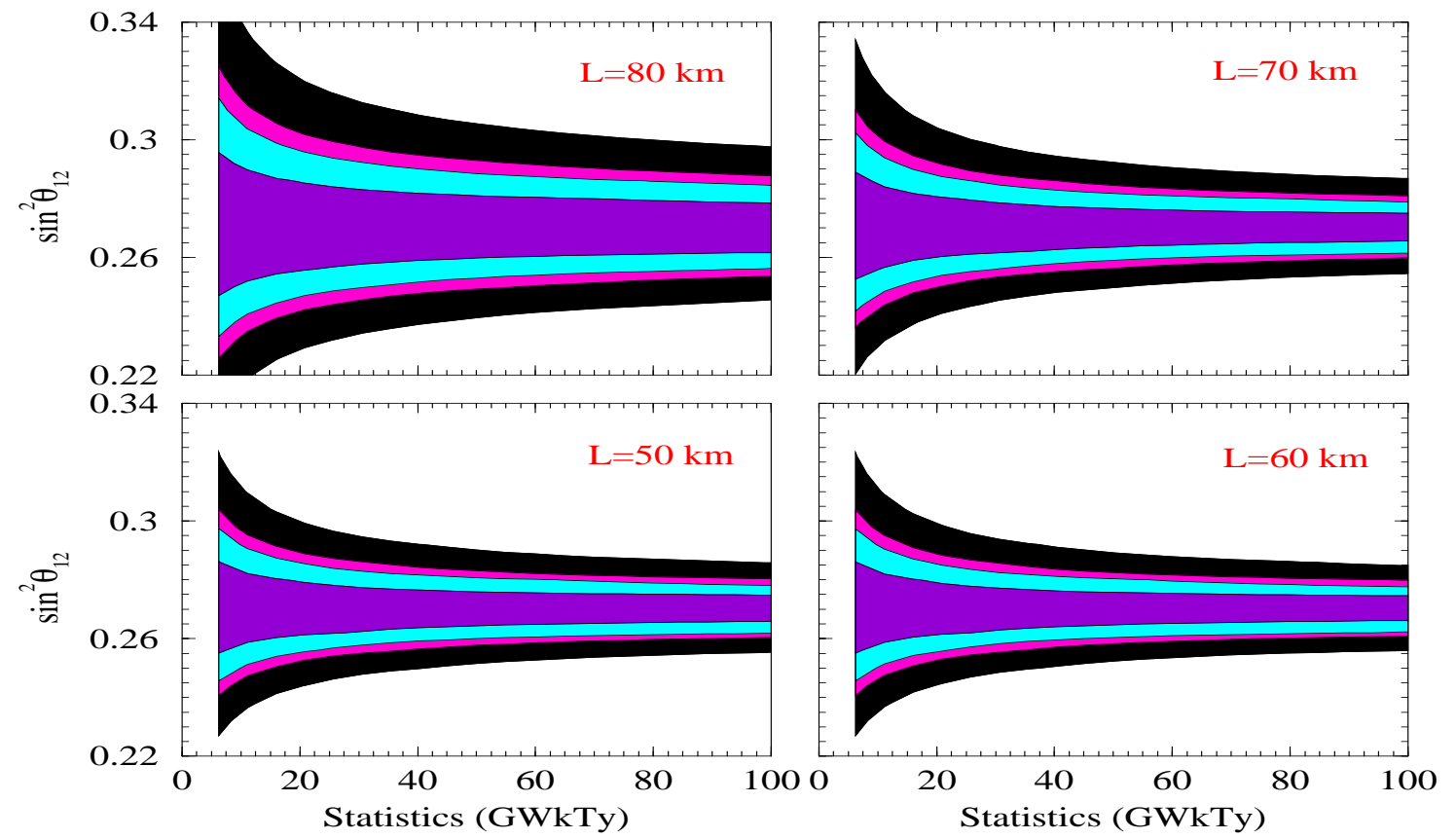

Figure 22: The precision on $\sin ^{2} \theta_{12}$ (at $68.27 \%, 90 \%, 95 \%, 99.73 \%$ C.L.) achievable in a second generation long baseline reactor neutrino experiment as a function of the statistics. Each of the panels corresponds to a certain fixed value of the baseline $L$. The results shown are for $\Delta m_{21}^{2}=8.3 \times 10^{-5} \mathrm{eV}^{2}$ and a true value of $\sin ^{2} \theta_{12}=0.27$ (from ref. [28]).

reactor fluxes, cross sections, and efficiencies would be substantially reduced.

In Fig. 22 [28] we show the sensivity on $\sin ^{2} \theta_{12}$ expected in a second generation long baseline reactor experiment which is tuned to the SPMIN [26]. In these plots we assume that the true value of $\sin ^{2} \theta_{12}=0.27$ and $\Delta m_{21}^{2}=8.3 \times 10^{-5} \mathrm{eV}^{2}$. We simulate the prospective data in a future long baseline reactor experiment tuned to SPMIN and use these data to obtain the measured value of $\sin ^{2} \theta_{12}$ in such an experiment. The measured value of $\sin ^{2} \theta_{12}$ is expected to be a band around the true value, with a certain uncertainty. The $68.27 \%$, $90 \%, 95 \%, 99.73 \%$ C.L. range of uncertainty in the measured value of $\sin ^{2} \theta_{12}$, expected in the proposed reactor experiment, is plotted in Figure 22 as a function of the statistics. The experimental statistics is given in units of GWkTy, which corresponds to the total reactor thermal power in units of GW times the total exposure of the detector in units of kTy. The total systematic uncertainty is assumed to be $2 \%$ and a prompt energy cut of $2.6 \mathrm{MeV}$ is imposed to avoid the geo-neutrino background. The 4 panels in Fig. 22 present the situation for 4 given baselines. We note that for a baseline of $L=60 \mathrm{~km}$, and a statistics of 20 GWkTy, the value of $\sin ^{2} \theta_{12}$ can be measured in the discussed experiment with an error of $\sim 3 \%(\sim 10 \%)$ at $1 \sigma(3 \sigma)$ level. The error in the measured $\sin ^{2} \theta_{12}$ would be reduced to 
$\sim 2 \%(\sim 6.5 \%)$ at $1 \sigma(3 \sigma)$, when the statistics is increased to 60 GWkTy. It should be noted that the currently existing $3 \sigma$ limit on $\sin ^{2} \theta_{13}[23,29], \sin ^{2} \theta_{13}<0.05$, implies an additional uncertainty in the measured value of $\sin ^{2} \theta_{12}$ which does not exceed approximately (2-3)\% $[30]$.

\subsection{Sterile Neutrinos}

Reactor experiments have the potential to explore and constrain the existence of sterile neutrinos. The presence of sterile neutrinos in addition to the three active flavors has been considered in order to explain the LSND signal together with the other solar, atmospheric, accelerator and reactor data.

Mixing of the electron neutrino with a sterile state would affect the disappearance of $\bar{\nu}_{e}$ in reactors.

The survival probability of $\bar{\nu}_{e}$ at nuclear reactors is given by:

$$
P\left(\bar{\nu}_{e} \rightarrow \bar{\nu}_{e}\right)=1-4 \sum_{a>b}\left|U_{1 a}\right|^{2}\left|U_{1 b}\right|^{2} \sin ^{2}\left(\frac{\Delta m_{a b} L}{4 E}\right)
$$

For a baseline around $1 \mathrm{~km}$, the mass splitting relevant for atmospheric neutrinos, $\Delta m_{\text {atm }}^{2}=$ $m_{3}^{2}-m_{1}^{2} \simeq 2 \times 10^{-3}$, gives the dominant contribution in the three flavors case. In the presence of a sterile neutrino with some not too small mixing with $\nu_{e}$, this is corrected by the contribution of the new $\Delta m_{s}^{2}=m_{4}^{2}-m_{1}^{2}$, if this is in the right range:

$$
P\left(\bar{\nu}_{e} \rightarrow \bar{\nu}_{e}\right) \approx 1-\sin ^{2} 2 \theta_{13} \sin ^{2}\left(\frac{\Delta m_{a t m} L}{4 E}\right)-\sin ^{2} 2 \theta_{s} \sin ^{2}\left(\frac{\Delta m_{s} L}{4 E}\right)
$$

If $\Delta m_{s}^{2}$ is $\sim 1 \mathrm{eV}$, as motivated by trying to accommodate the LSND experiment with the rest of the neutrino data, the $\Delta m_{s}^{2}$ oscillations are averaged out over $\mathrm{km}$ distances and the last term has a small, constant contribution to the survival probability. The experiment would in this case get a measurement of $\theta_{13}$ if $\theta_{13} \gtrsim .01$. In order to distinguish a constant contribution in the energy spectrum, the mixing angle $\theta_{s}$ would have to be larger than what is required by the LSND data. The contribution of such a sterile neutrino in a reactor experiment would become measurable if a detector would be placed at a very short distance from the reactor $(\sim 10 \mathrm{~m})$. In this case the $\Delta m_{s}^{2}$ oscillation becomes the dominant one and the experiment would get a measurement of $\theta_{s}$ if $\sin ^{2} 2 \theta_{s} \gtrsim .01$.

Solar and atmospheric data do not favor the presence of a sterile neutrino, but still allow it at a few percent level. If $\Delta m_{s}^{2}$ is of the order of $\Delta m_{a t m}^{2}$, both $\Delta m^{2}$ 's would contribute to the survival probability in a reactor experiment and it would be hard to measure them separately. This would be possible if the mass scales are still sufficiently different to give a 
significant distortion in the energy spectrum. If no oscillation effect is observed, the mixing angle limit obtained would apply to each of the two mixing angles, $\theta_{13}$ and $\theta_{s}$.

It would also be possible to constrain the existence of a sterile neutrino by adding reactor data to all the rest of the neutrino data and trying to perform an "unitarity check" for a three flavor mixing matrix. This would be possible with a combination of precise disappearance and appearance experiments and checking if $P\left(\nu_{e} \rightarrow \nu_{e}\right)+P\left(\nu_{e} \rightarrow \nu_{\mu}\right)+P\left(\nu_{e} \rightarrow \nu_{\tau}\right)$ adds up to 1.

\subsection{Tests of CPT Symmetry}

$\mathrm{CPT}$ is conserved quite generally in local relativitic quantum field theory, and even in large classes of non-local effective field theories. However, as far as is known, CPT is not gauged in string theory, and thus there is no general argument that it should be respected at high energies. Therefore, the test of this symmetry is a test of the way we understand high energy physics, and as such cannot be underestimated. CPT can be violated through a difference in the mass (or lifetime) of a particle-antiparticle pair or through decoherence. Both of these symmetry violations can be tested by a combination of reactor and solar neutrino data. Althoug CPT seems to be tested to an incredible level in the kaon sector, quoted as a limit on the possible differences in mass squared, these measurements give

$\left|m^{2}\left(K^{0}\right)-m^{2}\left(\bar{K}^{0}\right)\right|<.25 e V^{2}$, leaving room for considerable improvements in the neutrino sector.

\subsection{Supernova Neutrinos}

Although supernovae have been getting a lot of attention in recent years, the core collapse, which represents one of the most energetic events known, and the explosion, which ejects the envelope to outer space, are far from being understood. It is clear that the observation of neutrinos emitted from supernova will be essential to help us further our understanding of these fascinating objects.

State of the art models of neutrino emission are based on certain assumptions that do not stand on very firm ground. They are the following:

- Basically all the gravitational binding energy is emitted in the form of neutrinos.

- The characteristic emission time is approximately 10 seconds.

- The emitted energy is equally distributed among the six neutrino flavors.

- Due to the cross sections of the different flavors, the average energy of each flavor is not the same with $\left\langle E_{\nu_{e}}\right\rangle<\left\langle E_{\bar{\nu}_{e}}\right\rangle\left\langle\left\langle E_{\nu_{\mu}, \bar{\nu}_{\mu}, \nu_{\tau}, \bar{\nu}_{\tau}}\right\rangle\right.$ 
The detector for a reactor neutrino experiment has the potential to detect supernova neutrinos, not only through charged current processes (as the detectors containing free protons), but also through neutral current processes (by neutrino proton elastic scattering) being sensitive in this way to the flux of all active flavors. Therefore, if the last two assumptions are correct, one expects that the yield of the neutral current reactions will be dominated by non electron flavor neutrinos, which are responsible for $4 / 6$ of the total luminosity and have a higher energy and hence a bigger yield per particle making scintillator-based detectors complementary to (the much larger) water Cerenkov detectors.

Separate measurements of the total energy and temperature for each flavor (that can not be achieved in the Megaton size detectors) will provide a goldmine of useful information for testing supernova models and for comparing with numerical ones.

\subsection{Massive Anti-Neutrino Detectors for Monitoring Worldwide Reactors}

Some studies have been made [31] and a workshop held[32], considering huge electron antineutrino detectors, in the gigaton class, which might be employed to remotely monitor reactor activity around the world. In order to keep costs within credible limits it can be shown that such remote detectors must utilize water as the medium and be placed in the deep ocean or lakes. Cherenkov radiation does not provide enough signal for the inverse beta decay tagging, so some doping of the water is needed, as with a wavelength shifter. The ocean construction and handling problems are considerable but manageable. The largest technical/cost barrier is the photodetection system. Traditional photomultiplier costs would totally dominate. Perhaps a factor of ten can be gained by automated production lines. A better long range prospect may be new generation photodetectors, of a type envisaged as photo-detecting wallpaper, and employing the developing GEM technology and flexible circuit manufacturing techniques coupled with organic photocathode materials [32]. With technical development such 10 megaton deep ocean units might be manufactured for costs in the $\$ 100 \mathrm{M}$ range, in large numbers.

An array of such detectors in the world's oceans could detect clandestine reactors operating at levels that could make nuclear bomb material on a one-year timescale (25MWt). Moreover a large dispersed array of such detectors could detect clandestine fission bomb testing down to the less than kiloton TNT equivalent scale, anywhere in the world.

Such instruments, even the prototype, would have wonderful scientific spin-off [33]. For example one might push nucleon decay limits to $\geq 10^{36}$ years, severely testing SUSY. A gigaton instrument would see collapse supernovae out to well beyond the Virgo cluster (50Mpc), achieving rates of ten event signals as often as one per 5 days (SN rate very uncertain). In fact the rate of $\geq 6 \mathrm{MeV}$ events from the sum of all past SN would be 250-1000/day in 
such a detector. A $10 \mathrm{kpc}$ distant galactic SN would yield a blistering 250 million events, allowing detailed studies of supernova dynamics and neutrino properties. An increase in single neutron flux might also be detected, providing a several day warning of a supernova from anywhere in the galaxy as a massive star enters the silicon burning phase. Solar neutrino fluxes could be monitored for temporal variations, diurnal variations studied, etc., to heretofore undreamed of precision.

A possible significant background for such an instrument is from a hypothetical natural reactor at the earth's core, which drives the earth's magnetic field. A first step would be to study this possibility.

The scientific cornucopia from such an instrument would be so great that it would attract a large scientific community, addressing a serious concern of the defense related world of attracting top quality scientists to ongoing programs. And it would move so many orders of magnitude into unexplored territory that discovery seems inevitable. This is not a program that can be realized immediately. The intent of the exercise was to see where we may go in the next years or decades. That seems to be a very promising direction for new neutrino research and application to real world problems.

\subsection{A Possible Reactor at the Center of the Earth}

There has been a long-standing controversy over the $~ 44$ TW heat flow from the earth [34], and it is generally thought that there must be a source of heat inside the core boundary [35]. J. Marvin Herndon, a nuclear geochemist, has proposed [36, 37, 38] that there is a georeactor operating at the earth's center, and it generates the heat (3-10 TW) required to drive plumes which result in the earth's magnetic field. The poisoning of this breeder reactor results in quasi-periodic shutdown, and chaotic field reversals after restart, with the geologically surprising timescale of only $\sim 250,000$ years.

Perhaps the most compelling geological evidence for this hypothesis is the anomalously high ratio of ${ }^{3} \mathrm{He} /{ }^{4} \mathrm{He}$, that is found in mid-ocean volcano emissions, such as in Hawaii [38]. This the ratio ranges from 8 to 30 times that found in the atmosphere. ${ }^{3} \mathrm{He}$ is only produced from Tritium decay, and Tritium comes from nuclear fission. There are other peculiarities in isotope ratios from the earth as well.

However, most geologists have thought that the Uranium in the earth was largely in the crust, as it floated up as slag when combined with oxygen. Early on when there was more ${ }^{235} U$ there indeed were some natural reactors near Oklo in Africa. But whether there is $\mathrm{U}$ in the earth's core has to do with the initial mix and how much oxygen was present. Herndon challenges the standard assumptions which are largely based upon what we see at the earth's surface, which is similar to typical meteorites. He draws upon evidence from a rarer class of meteors (enstatite chondrites) which he argues are a better match to the earth's core 
properties [39].

There is some suggestive evidence from studies of other planets as well, which indicate that some of the gas giants radiate twice as much heat as can be explained by traditional means. Magnetic fields, atmospheres, and indeed even life on earth may be intimately intertwined with this planet-centered heat source.

Studying the putative georeactor directly is not feasible (except perhaps in a bold proposal by David Stevenson of CalTech [40]). One can however look for the electron antineutrinos which would present an unambiguous signature [41].

Interest in the possibility of a georeactor has grown, perhaps fueled somewhat by the recollection of the geology community's slow awakening to continental drift. There have been discussions amongst various groups around the world on how best to test the hypothesis directly with electron anti-neutrino detection. This was reported at a workshop in Hawaii in February 2004 [43, 44]. If a georeactor is present, it would present the major background for hypothetical giant anti-neutrino detectors for monitoring the world's reactors and keeping watch for clandestine bomb testing [42]. Also, groups in Russia [46] and the Netherlands [45] have posted manuscripts describing possible experiments. Clearly the best locations for such a measurement are far from man-made power reactors, and away from the continental plates which have high radioactivity. Depth is also a requirement, since cosmic ray muons make spallation products which cause dead-time and can make fake signatures. About $2 \mathrm{~km}$ water equivalent depth is adequate, though the deeper the better, either in a mine or underwater (or even ice). An experiment of the size of KamLAND (1 kiloton liquid scintillator) would make definitive measurements in several years (limit less than $1 \mathrm{TW}$ ). In the event of a positive signal, the small amount of directionality in such events can make positive statistical identification of the events as coming from the earth's core.

\section{Conclusion}

The worldwide program to understand neutrino oscillations and determine the mixing parameters, CP violating effects, and mass hierarchy will require a broad combination of measurements. Our group believes that a key element of this future neutrino program is a multi-detector neutrino experiment (with baselines of $200 \mathrm{~m}$ and $1.5 \mathrm{~km}$ ) with a sensitivity to $\sin ^{2} 2 \theta_{13}$ of 0.01 at $90 \%$ confidence level. In addition to oscillation physics, the reactor experiment may provide interesting measurements of $\sin ^{2} \theta_{W}$ at $Q^{2}=0$, neutrino couplings, magnetic moments, and mixing with sterile neutrino states.

The parameter $\theta_{13}$ is one of the twenty six parameters of the standard model, the best model of electroweak interactions for energies below $100 \mathrm{GeV}$ and, as such, is worthy of a precision measurement independent of other considerations. A reactor experiment of the 
proposed sensitivity will allow a measurement of $\theta_{13}$ with no ambiguities and significantly better precision than any other proposed experiment, or will set limits indicating the scale of future experiments required to make progress. A reactor experiment with a $1 \%$ precision may also resolve the degeneracy in the $\theta_{23}$ parameter (when combined with long-baseline accelerator experiments.)

In combination with long-baseline measurements, a reactor experiment may give early indications of $\mathrm{CP}$ violation and the mass hierarchy. The combination of the T2K and Nova long-baseline experiments will be able to make significant measurements of these effects if $\sin ^{2} \theta_{13}>0.05$ and with enhanced beam rates can improve their reach to the $\sin ^{2} \theta_{13}>0.02$ level. If $\theta_{13}$ turns out to be smaller than these values, one will need other strategies for getting to the physics. Thus, an unambiguous reactor measurement of $\theta_{13}$ is an important ingredient in planning the strategy for the future neutrino program.

\section{References}

[1] M. Apollonio et al., hep-ex/0301017

[2] C. Bemporad, G. Gratta, P. Vogel, Rev. Mod. Phys. 74 (2002) 297.

[3] P.Huber et al., hep-ph/0303232

[4] K. Anderson et al., hep-ex/0402041

[5] M. H. Shaevitz and J. M. Link arXiv:hep-ex/0306031, (2003).

[6] T. K. Gaisser and T. Stanev, Phys. Lett. B592, 228 (2004).

[7] Y. F. Wang, V. Balic, G. Gratta, A. Fasso, S. Roesler and A. Ferrari, Phys. Rev. D 64, 013012 (2001) [arXiv:hep-ex/0101049].

[8] T. Hagner, R. von Hentig, B. Heisinger, L. Oberauer, S. Schonert, F. von Feilitzsch and E. Nolte, Astropart. Phys. 14, 33 (2000).

[9] P.Huber et al., hep-ph/0403068

[10] http://braidwood.uchicago.edu/

[11] http://theta13.lbl.gov/

[12] http://www.hep.anl.gov/minos/reactor13/Angra/proposal.html

[13] http://doublechooz.in2p3.fr/ 
[14] Loinaz, W., N. Okamura, T. Takeuchi and L.C.R. Wijeewardhana, Phys. Rev. D 67 (2003) 07012.

[15] G. P. Zeller et al., Phys. Rev. Lett., 88 091802, 2002.

[16] Marciano, W.J. and Z. Parsa, hep-ph/0403168

Imlay, R. and G.J. VanDalen, J. Phys. G: Nucl. Part. Phys. 29(2003) 2647.

[17] J.M. Conrad, J.M. Link and M.H. Shaevitz, hep-ex/0403048.

[18] Mohapatra, R.N. and S-P Ng, hep-ph/0404274.

[19] Li, H.B. et al., Phys. Rev. lett. 90(2003)131802.

Daraktchieva, Z. et al., Phys. lett. B 564 (2003) 190.

Liu, D.W. et al., hep-ex/0402015.

[20] J. N. Bahcall and C. Peña-Garay, JHEP 0311, 004 (2003).

[21] K. Eguchi et al. [KamLAND Collaboration], Phys. Rev. Lett 90 (2003) 021802.

[22] T. Araki et al. [KamLAND Collaboration], hep-ex/0406035.

[23] A. Bandyopadhyay et al., hep-ph/0406328, version 2.

[24] A. Bandyopadhyay, S. Choubey, S. Goswami and S. T. Petcov, Phys. Lett. B581 (2004) 62.

[25] S. Choubey and S. T. Petcov, Phys. Lett. B 594, 333 (2004).

[26] A. Bandyopadhyay, S. Choubey and S. Goswami, Phys. Rev. D67 (2003) 113011.

[27] S.N. Ahmed et al., Phys. Rev. Lett. 92, 181301 (2004).

[28] S. Choubey, talk at NuFact'04, Osaka, Japan, 2004.

[29] J.N. Bahcall, M.C. Gonzalez-Garcia and C. Peña-Garay, hep-ph/0406294.

[30] S. Choubey, S. T. Petcov and M. Piai, Phys.Rev. D68 (2003) 113006.

[31] White paper for a gigaton array by J.G. Learned, see NACW proceedings below. Also talk at NU2004 Paris, 15 June 2004.

[32] Neutrinos and Arms Control Workshop, Honoulu 4-7 Feb 2004, http;//www.phys.Hawaii.edu/ jgl/nacw.html

[33] S. Pakvasa at NACW, above. 
[34] V. Dehant, et al., Earth's Core: Dynamics, Structure, Rotation (AGU, Washington DC, 2003) 277 pages.

[35] S. Scandolo, and R. Jeanloz, "The Centers of Planets," American Scientist 91: 516-525 (November-December 2003).

[36] J.M. Herndon, "Planetary and protostellar nuclear fission: implications for planetary change, stellar ignition and dark matter," Proceedings Royal Society London A 445: 453-462 (1994).

[37] D.F. Hollenbach and J.M. Herndon, "Deep-earth reactor: nuclear fission, helium, and the geomagnetic field", Proc. Nat. Acad. (USA) 98, 11085 (2001).

[38] J.M. Herndon, "Nuclear georeactor origin of oceanic basalt 3He/4He, evidence, and implications," Proceedings National Academy of Sciences 100: 3047-3050 (2003).

[39] J.M. Herndon, "The chemical composition of the interior shells of the earth", Proc. Roy. Soc.

[40] D.J. Stevenson, “A Modest Proposal: Mission to Earth's Core”, Nature (May 15, 2003).

[41] R. S. Raghavan, "Detecting a Nuclear Fission Reactor at the Center of the Earth", hep-ex/0208038;

[42] J.G. Learned, "Neutrinos and Arms Control: Thinking Big about Detection of Neutrinos from Reactors at Long Distances", see NACW proceedings below. Also talk at NU2004 Paris, 15 June 2004.

[43] E. Guillian, "Anti-neutrino Detection, Earth Reactor", unpublished at NACW website.

[44] Jelena Maricic, "Using KamLAND Data to Seek a Georeactor", PhD dissertation at U. Hawaii, in preparation (2004).

[45] R.J. de Meijer, E.R. van der Graaf, K.P. Jungmann, "Quest for the Nuclear Georeactor", accepted for Nuclear Physics News, 2004; physics/0404046

[46] G. Domogatski, V. Kopeikin, L. Mikaelyan, V. Sinev, "Neutrino Geophysics at Baksan I: Possible Detection of Georeactor Anti-neutrinos", Submitted to Physics of Atomic Nuclei; hep-ph/0401221 